\title{
La Constitution congolaise du 6 novembre 2015
}

Entre influence française et affirmation identitaire

The November 6, 2015 Constitution from Congo. Between French influence and affirming one's identity

\section{Vivien Romain Manangou}

\section{(2) OpenEdition}

\section{Journals}

Édition électronique

URL : http://journals.openedition.org/add/1566

DOI : $10.4000 /$ add. 1566

ISSN : 2606-1988

\section{Éditeur}

Presses universitaires de Rouen et du Havre

\section{Édition imprimée}

Date de publication : 1 mai 2019

Pagination : 121-159

ISBN : 979-10-240-1293-3

ISSN : 1955-0855

\section{Référence électronique}

Vivien Romain Manangou, «La Constitution congolaise du 6 novembre 2015 », Les Annales de droit [En ligne], 13 | 2019, mis en ligne le 09 décembre 2019, consulté le 24 janvier 2021. URL : http:// journals.openedition.org/add/1566; DOI : https://doi.org/10.4000/add.1566 


\title{
La Constitution congolaise du 6 novembre 2015
}

\author{
Entre influence française et affirmation identitaire
}

\author{
Vivien Romain Manangou
}

À travers le constitutionnalisme dit de la troisième génération ${ }^{1}$, JeanMarie Breton considère que l'Afrique «a lentement trouvé, au fil des années, les ferments de son originalité, les assises de sa légitimité, les conditions de son efficacité, et les forces de sa créativité ${ }^{2}$ ». Il est vrai que depuis quelques années, sur le continent de Mandela, les États brillent par une inventivité constitutionnelle, dont l'objectif est de fabriquer un modèle constitutionnel adapté aux réalités culturelles africaines. Mais de l'autre côté, il est impossible d'ignorer la persistante prégnance du modèle constitutionnel français ${ }^{3}$, ou pour le dire comme le professeur Dodzi Kokoroko, «le cycle constitutionnel reste tiraillé entre l'universalisme des mœurs politiques et le ghetto des particularismes culturels $^{4}{ }$. Ce couple volonté identitaire et influence exogène est bien illustré dans la nouvelle constitution de la république du Congo. Qu'on s'en réjouisse ou qu'on s'en exaspère, il faut admettre, comme le notait Yves Mény ${ }^{5}$, à propos de la France, que la République du Congo est une

1. La chronologie divisant le constitutionnalisme africain en trois âges est communément acceptée. Le $1^{\text {er }}$ âge correspond aux constitutions de la première génération des indépendances aux années 1970. Le $2^{\mathrm{e}}$ âge se rapporte au présidentialisme dit «négro-africain», un retour vers une identité africaine et donc nationaliste (1970). Enfin, le $3^{\mathrm{e}}$ âge est celui issu de la vague des conférences nationales des années 1990, avec le retour à un constitutionnalisme libéral.

2. Jean-Marie Breton, «Trente ans de constitutionnalisme d'importation dans les pays d'Afrique noire francophone entre mimétisme et réception critique: cohérences et incohérences (1960-1990)", AFDC, Congrès de Montpellier, 9-11 juin 2005, p. 1.

3. Jean Du Bois de Gaudusson, «Sur l'attractivité du modèle de la Constitution de 1958 en Afrique, cinquante ans après... », dans Bertrand Mathieu (dir.), Cinquantième anniversaire de la Constitution française, Paris, Dalloz, 2008, p. 675 et suiv.

4. Dodzi Kokoroko, «L'idée de Constitution en Afrique », Afrique contemporaine, vol. 2, $\mathrm{n}^{\mathrm{o}} 242,2012$, p. 117.

5. Yves Mény, «Des mœurs irréformables?», Pouvoirs, ${ }^{\circ}$ 126, 2008, p. 38. 
« dévoreuse de Constitutions consommées à la va-vite ${ }^{6}$ ». Comment en est-on venu au Congo en 2015 à élaborer un nouveau texte fondamental ? Comment a-t-elle été écrite et adoptée? Dans le fond, marque-t-elle véritablement une rupture avec sa devancière ${ }^{7}$ ? Telles sont les questions que j'ai souhaité aborder dans cette analyse. Tout d'abord, et à coup sûr, il convient d'évoquer le contexte dans lequel a été adoptée la Constitution de 2015.

Avec une rapidité inouïe, la Constitution du 20 janvier 2002, dont certains prétendaient qu'elle reflétait «la volonté des constituants d'établir les fondements d'une société nationale et démocratique ${ }^{8}$ ", était balayée - peut-être pour répondre à ce que le doyen Godefroy Moyen qualifiait « d'aspiration légitime des peuples d'Afrique [du Congo] à un nouvel ordre politique qui leur garantit [...] la liberté et la sécurité ${ }^{9} »-$ remplacée par une Constitution, qui d'après Collinet Makosso est plus «normative, non mimétique et nominaliste ${ }^{10}$ ».

Outre le débat animé sur l'opportunité et la légalité du changement de constitution ${ }^{11}$, tout commence véritablement avec les consultations organisées par le chef de l'État, portant notamment sur l'avenir des institutions ${ }^{12}$. Pendant une dizaine de jours, le président de la République consulte les forces vives de la Nation congolaise, à l'issue, il prononça un

6. La Constitution du 6 novembre 2015 est la $8^{\mathrm{e}}$ Constitution de la jeune histoire de la tout aussi jeune République congolaise. Sans compter les textes fondamentaux exceptionnels comme en 1991, après la conférence nationale, ou encore en 1997, après la guerre civile.

7. Avant l'adoption de la Constitution de 2015, la République du Congo était régie par la Constitution de 2002, adoptée à la fin de la transition flexible de cinq ans (1997-2002). Cette Constitution instaurait un régime présidentiel.

8. Guy Jean-Clément Mebiama, Le régime politique de la république du Congo après la Constitution de 2002, Paris, L'Harmattan, 2005, p. 52.

9. Godefroy Moyen, «L'exécutif dans le nouveau constitutionnalisme africain : le cas du Congo, du Bénin et du Togo", Les Annales de l'université Marien-Ngouabi, vol. 10, $\mathrm{n}^{\mathrm{o}} 3,2009$, p. 46.

10. Anatole Collinet Makosso, Le régime consensualiste dans la Constitution congolaise du 25 octobre 2015 commentée article par article, Brazzaville, L'Harmattan-Congo, 2016, p. 50.

11. Claude-Richard M’bissa, Débat sur la Constitution du 20 janvier 2002 au Congo, enjeux sociaux et stratégies politiques, Paris, L'Harmattan-Congo, 2014. Vivien Romain Manangou, "Plaidoyer pour une réforme radicale de la Constitution", La Semaine africaine, 27 août 2013, http://lasemaineafricaine.net/index.php/pointde-vue/6453-plaidoyer-pour-une-reforme-radicale-de-la-constitution.

12. Entre fin mai et début juin 2015, le chef de l'État avait organisé une consultation républicaine, pour s'entretenir avec les différentes forces vives de la nation congolaise. Ainsi, plus de 400 concitoyens avaient été consultés sur la vie de la nation et de l'État. Cependant, une partie de l'opposition et certains membres de 
discours à la télévision nationale pour convoquer un dialogue et y fixer les grandes lignes ${ }^{13}$.

À cette occasion, il annonça la mise en place d'une commission préparatoire au dialogue ${ }^{14}$, tout en soumettant des questions juridiques dont le comité d'experts de la commission devrait apporter des réponses. Ainsi, il revenait à la commission de répondre aux deux questions suivantes : comment devrait-on organiser au mieux les scrutins à venir dans notre pays? Peut-on, oui ou non, faire évoluer les institutions de la République? Si oui dans quel sens? Et si non, pourquoi ?

C'est donc pour répondre à ces deux questions qu'un document d'orientation avait été élaboré par la commission préparatoire, afin de le soumettre au débat lors du dialogue ${ }^{15}$. Il faut souligner au passage qu'une partie non négligeable de la classe politique et de la société civile s'opposait à ce dialogue, en avançant deux arguments, le premier d'ordre constitutionnel et le second d'ordre politique. Sur le plan constitutionnel, les opposants au dialogue ${ }^{16}$ arguaient qu'une telle initiative avait pour principal objectif de lever l'ensemble des verrous constitutionnels, empêchant une nouvelle candidature du chef de l'État sortant ${ }^{17}$. Pour ce qui est de l'argument politique, il s'agissait d'un argument d'opportunité, on ne pouvait pas, d'après eux, «changer les règles du jeu en cours de partie ou plutôt à quelques mois de l'échéance

la majorité présidentielle, opposés au changement de Constitution, avaient boudé ce rendez-vous.

13. Il s'exprima le 30 juin 2015 à la télévision. Le dialogue national fut convoqué du 13 au 15 juillet à Sibiti, dans le département de la Lékoumou, au sud du Congo.

14. D. $\mathrm{n}^{\mathrm{o}} 2015-660,1^{\mathrm{er}}$ juillet 2015 , portant nomination des membres de la commission préparatoire au dialogue national 2015.

15. Dialogue national, Commission préparatoire, «La réforme des institutions de la République », juillet 2015.

16. Les opposants au dialogue de Sibiti, regroupés principalement autour du Front pour le respect de l'ordre constitutionnel, l'alternance et la démocratie (FROCAD) et de l'Initiative pour la démocratie congolaise (IDC), ont boycotté le dialogue qualifiant la démarche de coup d'État institutionnel. Ils avaient d'ailleurs organisé un dialogue alternatif pour opposer leurs propositions à celles de la majorité. Voir «Congo: les opposants réunis à Brazzaville pour un dialogue alternatif», RFI Afrique, 21 juillet 2015, http://www.rfi.fr/afrique/20150724-congo-brazzavilleopposants-reunis-dialogue-alternatif-contestation-troisieme-manda.

17. En effet, l'art. 57 de la Constitution de 2002 prévoyait qu'un président de la République ne pouvait faire plus de deux mandats. Élu en 2002, puis réélu en 2009, le président Sassou ne pouvait plus constitutionnellement briguer un troisième mandat. Quant à l'art. 58, il posait un âge maximal de 70 ans pour être candidat, or le chef de l'État sortant, né en 1943, était âgé de 73 ans en 2016. Enfin, et sans doute le plus contraignant, l'art. 185 verrouillait la voie de la révision pour toute initiative concernant notamment le nombre de mandats présidentiels. 
présidentielle, il fallait attendre après la présidentielle pour opérer une révision ou pourquoi pas un changement de Constitution».

"La Constitution, toute la Constitution, rien que la Constitution ${ }^{18}$ ", disait François Mitterrand, assurément, ce rappel lors de la cohabitation avait tout son sens au Congo. Malheureusement, comme l'observait Pierre Avril, «la politique ne perd pas ses droits ${ }^{19}$ », et au nom sans doute du fidéisme de Jouvenel qui implique que, «la source légitime du pouvoir supplante l'emploi légitime du pouvoir ${ }^{20}$ ", la majorité présidentielle avançait, convaincue que la majorité des Congolais avançaient avec elle ${ }^{21}$. En effet, pour Philippe Lauvaux, «le fidéisme démocratique consiste, à l'intérieur du système démocratique [...] libéral, à poser le primat de la source démocratique du pouvoir sur le contenu libéral des normes [...], l'essentiel est que soit obéie la volonté de la majorité $[\ldots]^{22} »$. Ainsi se crée une fiction qui dicte l'action des gouvernants, se préoccupant peu du contenu et des exigences en la matière, ce qui compte c'est essentiellement la volonté de la majorité. André Laignel semble être celui qui avait le mieux résumé cette théorie, lorsqu'il répondit à Jean Foyer à l'Assemblée nationale : «vous avez juridiquement tort, parce que vous êtes politiquement minoritaire ${ }^{23}$.»

Le dialogue national ${ }^{24}$ adopta dans un curieux consensus ${ }^{25}$ le principe d'une évolution des institutions par le changement de la Constitution. L'objet de la réforme constitutionnelle était donc clair. Il s'agissait

18. Message au Parlement français, 8 avril 1986.

19. Pierre Avril, «Enchantement et désenchantement constitutionnels sous la $\mathrm{V}^{\mathrm{e}}$ République ", Pouvoirs, $\mathrm{n}^{\mathrm{o}}$ 126, 2008, p. 11.

20. Bernard de Jouvenel, De la souveraineté, Paris, Librairie de Médicis, 1955, p. 8.

21. Dans son allocution à la nation du 30 juin 2015, le chef de l'État affirma: «Audelà des nuances et des divergences, l'unanimité s'est formée autour de l'urgente nécessité d'aller à un dialogue national sans exclusive, sans préalable, sans a priori, afin que, dans la confiance et la sérénité, se dégage un indispensable consensus sur les questions à l'origine de profonds désaccords au sein de l'opinion nationale. » Voir aussi «L'intérêt supérieur de la nation triomphe à Sibiti », Le Patriote [hebdomadaire d'information], $\mathrm{n}^{\circ} 340,20$ juillet 2015, p. 2.

22. Philippe Lauvaux, Les grandes démocraties contemporaines, Paris, PUF, 2004, p. 49.

23. André Laignel à Jean Foyer, Assemblée nationale, 13 octobre 1981, cité dans JeanJacques Becker et Pascal Ory, Crises et alternances, 1974-200o, Paris, Seuil, 2002.

24. Convoqué par le Décret présidentiel $n^{0}$ 2015-691 du 9 juillet 2015 portant convocation du dialogue national 2015.

25. Dans sa déclaration devant les 629 délégués au dialogue, M. Firmin Ayessa, alors directeur de cabinet et représentant personnel du chef de l'État, affirma: «Pas de diatribe. Pas d'invectives. Pas de chahut. Pas de diktat. Pas de vote. Le consensus rien que le consensus", Communiqué final du dialogue national sans exclusive à Sibiti, le 17 juillet 2015 . 
d'abord et avant tout de répondre à la crise institutionnelle, comprendre, neutraliser les verrous constitutionnels empêchant le chef de l'État sortant de solliciter un nouveau mandat. Puis «de concilier les valeurs universelles de la démocratie [avec] les réalités politiques, sociales et culturelles de notre pays ${ }^{26}{ }$. Comme pour la loi du 3 juin 1958 - dont Michel Debré estimait que l'élaboration de la Constitution française de 1958 avait scrupuleusement respecté l'esprit ${ }^{27}$ - les participants aux dialogues avaient posé une série d'orientations devant se retrouver dans la nouvelle constitution ${ }^{28}$. Une sorte d'encadrement matériel de la future loi fondamentale. Il est vrai, comme le soulignait Frédéric Rouvillois, que «le droit constitutionnel n'est au fond, à beaucoup d'égards, que la formulation juridique plus ou moins transparente de certaines options politiques $^{29}$ ". Ainsi, le projet de Constitution, rédigé par une équipe d'experts désignés par le chef de l'État ${ }^{30}$ reprenait, pour l'essentiel, les recommandations issues du dialogue national de Sibiti. Toutefois, c'est dans la tourmente, après quelques jours de violence et dans un calme

26. Ibid., p. 6.

27. Michel Debré, «La nouvelle Constitution», dans Michel Debré et Nicholas Wahl (dir.), Naissance de la Cinquième République. Analyse de la Constitution par la revue française de science politique en 1959, Paris, Presses de la fondation nationale des sciences politiques, 1959, p. 25.

28. Il s'agissait de : l'institutionnalisation des valeurs du dialogue et du partage; l'instauration d'un exécutif bicéphale par la création d'un poste de Premier ministre, nommé par le président de la République; la fixation de l'âge pour être éligible à l'élection présidentielle à 30 ans minimum; la fixation du mandat présidentiel à cinq ans renouvelable; la mise en place d'une gouvernance qui prend en compte les différentes composantes de la société congolaise, notamment les femmes, les jeunes, les personnes vivant avec un handicap et les populations autochtones; le renforcement des pouvoirs du Parlement sur le contrôle de l'action de l'exécutif, notamment par l'usage de la motion de défiance à l'encontre d'un membre du Gouvernement et de la motion de censure contre le Gouvernement; la reconnaissance d'un statut particulier de l'opposition; la reconnaissance du statut des anciens présidents de la République, des anciens présidents des chambres parlementaires et des anciens Premiers ministres; le renforcement de la décentralisation; la prise en compte des notabilités traditionnelles et religieuses; la prise en compte de la diaspora; la prise en compte des questions de protection et de préservation de l'environnement.

29. Frédéric Rouvillois, Droit constitutionnel, Paris, Flammarion, 2015, p. 17.

30. Le décret présidentiel $n^{\circ}$ 2015-915 du 21 septembre 2015 avait désigné le $P^{r}$ Placide Moudoudou, ancien doyen de la faculté de Droit de l'université Marien-Ngouabi (Brazzaville), à la présidence de la Commission d'élaboration de cette nouvelle constitution. Son successeur au poste de doyen, le $\mathrm{P}^{\mathrm{r}}$ Godefroy Moyen, y siégeait comme vice-président. 
précaire $^{31}$ que sera adoptée la nouvelle constitution par référendum le 25 octobre $2015^{32}$. Une fois de plus, c'est au prix du sang que la question constitutionnelle se règle au Congo ${ }^{33}$, sans doute la faute à ce «leadership décevant ${ }^{34}$ ", décrit par le professeur Koffi Ahadzi Nonou. Tout compte fait, on en revient au constat d'Esmein selon lequel, « dans toute constitution, on arrive fatalement à certains points où le respect de la loi a pour unique garantie la conscience des autorités chargées de l'appliquer ${ }^{35}$ ».

Pour l'essentiel, la Constitution ainsi adoptée consacre « les principes suprapositifs, les droits de l'homme et la séparation des pouvoirs, destinés à fixer des bornes aux pouvoirs ${ }^{36}{ }$. Une Constitution plutôt libérale, dont seule la pratique permettra de véritablement l'arrimer dans cette catégorie, étant entendu, comme le pensait Léon Duguit, que «l'étude du droit public isolée de l'histoire et des faits est vaine et artificielle ${ }^{37}$ ».

Nicholas Wahl disait que «toutes les constitutions combinent une critique des institutions politiques défuntes, une philosophie du pouvoir et un schéma particulier d'organisation gouvernementale ${ }^{38} »$. Difficile

31. Des manifestations de l'opposition furent interdites, des affrontements entre militants d'opposition et les forces de l'ordre causèrent des blessés, ainsi que des morts à travers le pays, notamment à Pointe-Noire, capitale économique du pays. Pour se faire une idée des tensions peu avant le référendum, lire: Joan Tilouine, "Brazzaville, une capitale divisée à la veille d'un référendum contesté», Le Monde, 24 octobre 2015, http://www.lemonde.fr/afrique/article/ 2015/10/24/brazzaville-une-capitale-divisee-a-la-veille-d-un-referendumconteste_4796338_3212.html\#T1tk2QEobOgKSvo6.99.

32. Le «oui» avait obtenu $92,96 \%$ des suffrages exprimés, a déclaré le ministre de l'Intérieur Raymond Mboulou lors d'une allocution à la télévision nationale, ajoutant que le «texte de nouvelle constitution [entrerait] en vigueur dès sa promulgation par le président de la République ». Selon la Commission électorale, le taux de participation a été de $72,44 \%$, alors que l'opposition estimait la participation autour de $10 \%$.

33. Déjà le 30 novembre 1992, des Congolais furent tués à la suite des désaccords sur l'interprétation de la Constitution du 15 mars 1992. Voir Patrice Yengo, La guerre civile au Congo-Brazzaville, Paris, Karthala, 2006.

34. Koffi Ahadzi Nonou, "L'Afrique face aux défis d'une nouvelle voie: le défi du leadership ", Au-delà du développement: changer de voie, 30 septembre 2010. L'auteur y décrit la déception des peuples de l'Afrique envers les élites africaines, incapables d'impulser le véritable développement du continent.

35. Adhémar Esmein, "Note de jurisprudence parlementaire», RDP, vol. 23, 1901, p. 314 .

36. Frédéric Rouvillois, op. cit., p. 15.

37. Léon Duguit, $R D P$, vol. 12, 1899, p. 526.

38. Nicholas Wahl, "Aux origines de la nouvelle Constitution", dans Michel Debré et Nicholas Wahl (dir.), op. cit., p. 50. 
de trouver des traces d'une quelconque critique des institutions issues de la Constitution du 20 janvier 2002, ni même une philosophie du pouvoir différente - les deux constitutions consacrent, bien qu'à des degrés différents, un présidentialisme paternaliste ${ }^{39}$-, c'est simplement au niveau de la structuration gouvernementale que les choses semblent changer avec l'institution du poste de Premier ministre.

Finalement, il paraît certain qu'une analyse complète des sources de ce nouveau texte fondamental ne sera possible que lorsque les auteurs de cette Constitution auront décidé de mettre à la disposition du public les documents relatifs. En attendant, sur la foi de certaines dispositions constitutionnelles, on peut assez facilement déceler l'influence du modèle constitutionnel français (1). Au demeurant, on sait aussi que le président de ladite commission accorde une importance particulière à ce qu'il qualifie de "constitutionnalisme identitaire ${ }^{40}$ ", une volonté de renouer avec une certaine identité constitutionnelle congolaise (2), ces deux aspects semblent donc incontournables.

\section{La prégnante influence du modèle constitutionnel français}

La France est peut-être une «démocratie à part ${ }^{41}$ », mais elle suscite toujours autant d'attirances, notamment auprès de ses anciennes colonies. De cette manière, l'une des tendances du constitutionnalisme africain ${ }^{42}$ demeure «l'attractivité toujours renouvelée de la Constitution de 1958 et par là du constitutionnalisme français ${ }^{43}$ ». Ainsi, l'étude de la Constitution congolaise du 6 novembre 2015 fait apparaitre une domination des sources françaises, avec la reprise de certaines dispositions de la Constitution du général de Gaulle (1.1). En même temps, comme le soulignait Jean Du Bois de Gaudusson, «le retour en force du modèle de la Constitution de 1958 auquel on assiste [...] s'effectue dans un

39. Vivien Romain Manangou, «Le néo-présidentialisme africain: entre paternalisme et superposition ", Revue française de droit constitutionnel, vol. 103, $\mathrm{n}^{\circ} 3,2015$, p. 26-53, https://www.cairn.info/revue-francaise-de-droit-constitutionnel-2015-3page-e26.htm.

40. Placide Moudoudou, «L'État africain : entre constitutionnalisme libéral et constitutionnalisme identitaire", Revue CAMES/SJP, $\mathrm{n}^{\circ}$ 2, 2015, p. 113-140.

41. Selon la formule d'Oliver Duhamel, «Une démocratie à part», Pouvoirs, vol. 126, $\mathrm{n}^{\mathrm{o}}$ 3, 2008, p. 17-26.

42. Koffi Ahadzi Nonou, «Les nouvelles tendances du constitutionnalisme africain : le cas des États d'Afrique noire francophone», Afrique juridique et politique, juilletdécembre 2002, p. 35-86.

43. Jean Du Bois de Gaudusson, «Sur l'attractivité du modèle de la Constitution de 1958 en Afrique, cinquante ans après... », dans Bertrand Mathieu (dir.), op. cit., p. 1. 
autre contexte ${ }^{44}$ ». De cette façon, bien que les phénomènes d'imitation décrits par le doyen Rivero ${ }^{45}$ restent valables, néanmoins, «la recherche et la production du meilleur système de gouvernement ${ }^{46}{ }$ ont conduit les constituants congolais à s'approprier certaines dispositions de la Constitution de 1958 pour mieux les tropicaliser (1.2).

\subsection{Des dispositions directement tirées de la Constitution française de 1958}

Affirmer avec certitude que des dispositions présentes dans la Constitution congolaise de 2015 seraient en réalité des copies des dispositions figurant dans la Constitution française de 1958 pose au moins une difficulté d'ordre épistémologique. Cette difficulté vient « du phénomène par lequel des mécanismes constitutionnels identifiés et spécifiquement associés à une Constitution ou un droit constitutionnel se retrouvent postérieurement dans le droit constitutionnel d'un autre État ${ }^{47}$ ». L'ambiguïté de la réception des modèles est connue ${ }^{48}$. Cependant, il est aisé d'identifier les dispositions de sources françaises tant la fonction présidentielle, les rapports avec le Gouvernement et avec le Parlement sont assez spécifiques sous la $\mathrm{V}^{\mathrm{e}}$ République ${ }^{49}$. De la même manière, il ne fait aucun doute que la France avait emprunté aux Américains l'idée d'un président de la République aux pouvoirs étendus. Il semble clair que la majesté du président congolais dans la Constitution du 6 novembre 2015 tire sa source dans la conception gaullienne des institutions. Un article incarne majestueusement cette inspiration française: c'est l'article 64, qui dispose que :

Le président de la République est le chef de l'État. Il est le garant de l'indépendance nationale, de l'intégrité du territoire et de l'unité nationale,

44. Ibid.

45. Jean Rivero, «Les phénomènes d'imitation des modèles étrangers en droit administratif », dans Mélanges W. J. Ganshof Van Der Meersch, Bruxelles, Bruylant; Paris, LGDJ, 1972, t. III.

46. Stéphane Bolle, "Des constitutions "made in" Afrique», Communication au $\mathrm{VI}^{e}$ Congrès français de droit constitutionnel, Montpellier, 9-11 juin 2005, p. 1.

47. Delphine Chalus, «L'importation du droit constitutionnel français par les États de la C.E.I. », ibid., p. 1.

48. Pierre-Xavier Boyer, La réception des modèles étrangers dans le droit constitutionnel français: Grande-Bretagne et États-Unis au prisme de la réflexion française (1789-1958), thèse, université de Rouen, 2002.

49. Selon la formule d’Evangélia Georgitsi («La spécificité de la $V^{\mathrm{e}}$ République et les classifications: une opposition fausse», Revue française de droit constitutionnel, vol. $83, \mathrm{n}^{\mathrm{o}} 3,2010$, p. 543-564. 
du respect de la Constitution, des traités et accords internationaux. Il détermine la politique étrangère et de défense de la Nation. Le président de la République est le garant du fonctionnement régulier des pouvoirs publics et de la continuité de l'État.

Cet article est une réplique de l'article 5 de la Constitution française de 1958, au moins dans son esprit. Il s'agit, pour reprendre la formule de Didier Maus, du « fondement de la prépondérance présidentielle ${ }^{50}$ ». Ainsi, l'article 64 est un condensé général des missions présidentielles et non des pouvoirs. Il s'agit d'une série de dispositions qui permettent au président de la République de prendre «les initiatives nécessaires pour que les pouvoirs publics puissent remplir leurs missions respectives dans l'intérêt de la nation et le respect de la [...] Constitution ${ }^{51}$ ». L'article 64 consacre donc une fonction présidentielle autonome, le centre névralgique du pôle exécutif dans l'architecture institutionnelle adoptée par le constituant de 2015. De cette manière, nous pouvons, à l'instar de Didier Maus pour ce qui est de la $\mathrm{V}^{\mathrm{e}}$ République ${ }^{52}$, distinguer deux catégories de compétences contenues dans cet article: les compétences ayant des incidences sur la politique interne, et celles ayant des incidences sur la politique externe.

\subsubsection{Les incidences de l'article 64 sur la politique externe}

Sur le plan externe d'abord, l'article 64 fait du chef de l'État, «le garant de l'indépendance», mais aussi celui des «traités et accords internationaux ». Il détermine par ailleurs, «la politique étrangère et de défense de la Nation».

Ainsi, il oblige le président de la République à prendre toutes les dispositions nécessaires pour maintenir l'indépendance du pays, que cela passe par la diplomatie ou encore par les armées (défense). De cette obligation découle une série de conséquences pratiques. En premier lieu, la diplomatie et la défense nationale relèvent de la compétence du président de la République, c'est le fameux «domaine réservé ${ }^{33}$ ».

50. Didier Maus, «La Constitution jugée par sa pratique. Réflexions pour un bilan », Revue française de sciences politiques, vol. 34, $\mathrm{n}^{0} 4-5,1984, \mathrm{p} .878$.

51. Guy Mollet, Quinze ans après. La Constitution de 1958, Paris, Albin Michel, 1973, p. 69.

52. Didier Maus, «La Constitution jugée par sa pratique», art. cité, p. 880. Nous reprenons la distinction de l'auteur entre les affaires intérieures de l'État et les affaires extérieures de ce dernier.

53. Jean Massot, La présidence de la République en France, Paris, La Documentation française, 1977. 
De ce fait, comme en France sous la $\mathrm{V}^{\mathrm{e}}$ République, «il nomme les ambassadeurs et les envoyés extraordinaires auprès des puissances étrangères et des organisations internationales. Les ambassadeurs et les envoyés extraordinaires étrangers sont accrédités auprès de lui » (article 89).

En second lieu, pour assurer la défense de la nation, il dispose de la force militaire dont il est le chef. À ce titre, «il préside le comité de défense ainsi que les organes supérieurs d'orientation, de suivi et de décision stratégique en matière de défense et de sécurité » (art. 90). La politique étrangère et la défense nationale constituent des missions traditionnelles de la fonction exécutive. Ainsi, d'autres textes fondamentaux attribuent cette compétence au Gouvernement ${ }^{54}$, ou mieux encore, au président de la République ${ }^{55}$. Cependant, nous savons que la $\mathrm{V}^{\mathrm{e}}$ République est allée très loin dans la répartition des compétences dans le domaine dit réservé, au point de créer une «controverse constitutionnelle ${ }^{56}$ ».

L'étude des compétences présidentielles dans les domaines régaliens dans la Constitution de 2015 laisse apparaître sans grande difficulté une proximité évidente avec le système institué par la $\mathrm{V}^{\mathrm{e}}$ République.

\subsubsection{Les implications de l'article 64 sur le plan interne}

Concernant le plan interne, la Constitution fait du président de la République «le chef de l'État», à qui elle confie la mission de faire « respecter la constitution». Enfin, il lui revient de garantir « [le] fonctionnement régulier des pouvoirs publics et [de] la continuité de l'État ». À l'unisson de la $\mathrm{V}^{\mathrm{e}}$ République, le texte fondamental congolais fait du président de la République un gardien de la Constitution ${ }^{57}$. La

54. Les art. 65 et 65 a de la fondamentale de la République fédérale d'Allemagne du 23 mai 1949 confie, au chancelier et aux ministres fédéraux de la Défense et des Affaires étrangères, la responsabilité de la conduite des deux domaines.

55. L'art. II-section 1 de la Constitution des USA du 17 septembre 1787 fait du chef de l'État le commandant en chef des armées et le principal décideur en matière de politiques étrangères.

56. Julien Thomas, «Controverse sur la répartition constitutionnelle des compétences en matière de Défense ", Communication au $\mathrm{VI}^{e}$ Congrès français de droit constitutionnel, déjà cité.

57. Le débat sur le gardien de la Constitution a donné lieu à une controverse entre Carl Schmitt et Hans Kelsen. Voir Carl Schmitt, Le gardien de la Constitution, Paris, Dalloz, 2015; Olivier Beaud et Pascale Pasquino, La controverse sur «le gardien de la Constitution » et la justice constitutionnelle: Kelsen contre Schmitt, Paris, PanthéonAssas, 2007. 
réalisation de cette mission débouche sur une série de pouvoirs confiés au président de la République. Ainsi, il lui revient de promulguer la loi, conformément à l'article 85. Concernant cet article, il convient de souligner les similitudes avec le système français. En effet, le président de la République dispose d'un délai de quinze jours pour promulguer la loi. Par ailleurs, il peut solliciter une seconde lecture dans l'une ou l'autre chambre sans qu'un refus lui soit opposé. La seule différence entre les deux articles est sémantique, l'article 10 de la Constitution française concerne le Parlement, alors que l'article 85 du Congo pointe l'une ou l'autre chambre du parlement.

De la même manière, l'article 64 attribue également au président de la République la mission de garantir «le fonctionnement régulier des pouvoirs publics et de la continuité de l'État». De cette manière, comme avec la Constitution gaulliste, la loi fondamentale congolaise donne au président des pouvoirs exceptionnels lui permettant de répondre en cas de crise conduisant à une discontinuité de l'État. Ainsi, à l'instar de la dictature romaine et de l'article 16 de la Constitution française de $1958^{58}$, l'article 93 de la Constitution congolaise de 2015 permet la confusion temporaire des pouvoirs pour répondre à la mise en danger des institutions.

L'essentiel des pouvoirs présidentiels, ainsi que ses missions peuvent se retrouver dans l'article 64, qui est donc une disposition matricielle. D'ailleurs, c'est toujours au nom de la garantie du fonctionnement régulier des pouvoirs publics que le chef de l'État dispose du droit de grâce de l'article 92, ou encore qu'il assure la présidence du Conseil supérieur de la magistrature, en vertu de l'article 91. Mais c'est surtout dans ses rapports avec le Gouvernement et le Parlement que la prépondérance présidentielle prend tout son sens. Toutefois, le constituant congolais de 2015 a voulu tropicaliser la copie pour mieux l'adapter aux réalités congolaises, et particulièrement dans les rapports entre le président de la République et le Gouvernement d'une part et dans ceux avec le Parlement d'autre part.

\subsection{Une influence nuancée par une volonté de dépassement du texte de 1958}

Selon Karl Loewenstein, il est fondamental de déterminer l'ontologie d'une Constitution, c'est-à-dire, «la recherche de la signification réelle

58. Mathieu Carpentier, "État d'exception et dictature», Tracés. Revue de sciences humaines, $\mathrm{n}^{\mathrm{o}} 20,2011$, http://traces.revues.org/5060. 
d'une Constitution écrite, à l'intérieur d'un milieu national spécifiquement, et plus particulièrement de sa réalité pour le citoyen moyen ${ }^{59}$ »; or une Constitution «copier-coller», pour parler comme Stéphane Bolle ${ }^{60}$, ne peut pas véritablement prendre en compte l'aspect ontologique décrit. Heureusement, dans le cadre du constitutionnalisme de la troisième génération, «s'entremêlent imitations et innovations ${ }^{61}$ ». Les États africains qui s'inspirent de la Constitution française de 1958 ne se limitent plus à une reproduction servile, au contraire, ils essaient de l'adapter aux réalités locales. De la sorte, les constituants congolais de 2015 ont essayé de dépasser le texte de 1958 de deux manières: soit en corrigeant ce qu'ils considéraient comme des ambiguïtés, soit en allant plus loin dans la rédaction de certaines dispositions. L'objectif était, comme le soulignait Collinet Makosso, de sortir de «l'hypocrisie française ${ }^{62}$ ».

\subsubsection{Le refus d'une dyarchie au sommet de l'État}

"Un exécutif fort est la condition technique d'une démocratie libre», constatait André Tardieu ${ }^{63}$. Dans l'esprit des constitutionnalistes gaullistes, le rétablissement de l'autorité de l'État passait par une mise en avant du pôle exécutif. «Il nous faut un régime dans lequel le pouvoir exécutif, le pouvoir législatif et le pouvoir judiciaire sont séparés de leurs attributions, leurs hommes et leurs responsabilités afin qu'il ait un vrai gouvernement ${ }^{64}$ ", précisait l'homme de Bayeux. La séparation des pouvoirs est ici pensée comme un instrument permettant d'atteindre l'efficacité gouvernementale. Cependant, on le sait, la $\mathrm{V}^{\mathrm{e}}$ République est le régime des paradoxes: alors que les articles 5 et 20 mettaient clairement en œuvre une dyarchie au sommet de l'État, le général de Gaulle, «seul à détenir et à déléguer l'autorité de l'État [...] contestait à ses Premiers ministres le titre de chef du Gouvernement et en déduisait

59. Stéphane Bolle, art. cité, p. 11.

60. Karl Loewenstein, «Réflexions sur la valeur des Constitutions dans une époque révolutionnaire. Esquisse d'une ontologie des Constitutions », Revue française des sciences politiques, vol. 2, $\mathrm{n}^{\circ} 1,1952, \mathrm{p} .8$.

61. Stéphane Bolle, art. cité, p. III.

62. Anatole Collinet Makosso, op. cit., p. 195.

63. André Tardieu, "L'agonie de l'exécutif» (sur le $1^{\mathrm{er}}$ avril 1933), cité par Stéphane Pinon, Les réformistes constitutionnels des années trente aux origines de la $\mathrm{V}^{e}$ République, Paris, LGDJ, 2003, p. 307.

64. Charles de Gaulle, Discours et messages (1946-1958), Paris, Plon, 1970, p. 123. 
qu'il n'existait pas de dyarchie au sommet de l'État ${ }^{65}$ ». Les constituants congolais ont considéré qu'il y avait là un possible sujet de discorde, et qu'il fallait imposer une lecture présidentialiste du régime non par la pratique, comme sous la $\mathrm{V}^{\mathrm{e}}$ République, mais en l'affirmant clairement dans le texte. Ainsi, la répartition des compétences entre le chef de l'État et le chef du Gouvernement se fait de façon précautionneuse, avec le souci de garantir la prééminence présidentielle. De cette manière, on assiste à l'émergence d'un Premier ministre-intendant ${ }^{66}$, sans réelle assise politique. La volonté de dépassement est davantage palpable lorsqu'on compare les dispositions concernant la répartition des compétences entre les deux têtes de l'exécutif.

Si le style rédactionnel semble être le même entre les articles 8 de la Constitution de 1958 et l'article 83 de la Constitution de 2015, les deux articles organisant la nomination et la démission du Gouvernement ${ }^{67}$, le constituant congolais a semblé vouloir répondre à une "impasse" existant dans la Constitution de $1958^{68}$. En effet, l'article 8 ne prévoit pas l'hypothèse où le chef de l'État déciderait de renvoyer, de son plein gré, le chef du Gouvernement. La seule possibilité ouverte étant de le faire démissionner, mais qu'arriverait-il si un Premier ministre s'accrochait à son poste et disposait d'une majorité au Parlement? Il est vrai que cette disposition conforte l'idée de la dyarchie, avec la neutralisation des deux chefs du pôle exécutif. Peut-être s'agit-il du résultat de l'impact des ministres d'État dans la rédaction de la Constitution de $1958^{69}$ ?

65. Jean Massot, La dyarchie à la tête de l'État: une spécificité de la $V^{e}$ République qu'interrogent encore les constitutionnalistes, Paris, La Documentation française, 2008.

66. Nous nous inspirons du terme de Thierry Moungalla, qui compare le travail du Premier ministre à «l'intendance», cité par Anatole Collinet Makosso, op. cit., p. 196.

67. Pour une vision globale de nomination des Premiers ministres dans les régimes semi-présidentiels, voir David J. Samuels et Matthew S. Shugart, «La nomination et la révocation du Premier ministre en régime semi-présidentiel : l'impact de la présidentialisation des partis ", Revue internationale de politique comparée, vol. 17, $\mathrm{n}^{\mathrm{o}} 1$, 2010, p. 67-91.

68. René Capitant considérait par exemple que la Constitution de 1958 est «le texte le plus mal rédigé de l'histoire constitutionnelle [française] ", dans Léo Hamon, De Gaulle dans la République [1958], repris dans Écrits constitutionnels, Paris, CNRS, 1982, « Préface», p. 14.

69. Pendant longtemps l'apport des ministres d'État, notamment de Guy Mollet avait été sous-estimé par les analystes de la chose constitutionnelle. Or ce fervent défenseur du parlementarisme pouvait très bien influencer cette neutralisation des chefs de l'exécutif. Voir, dans ce sens, Didier Maus, « Guy Mollet et l'élaboration de la Constitution de 1958", dans Bernard Ménager, Philipe Ratte et al. (dir.), Guy 
L'alinéa 1 de l'article 83 de la Constitution de 2015 lève quant à lui l'ambiguité: «Le président de la République nomme le Premier ministre et met fin à ses fonctions. » Dans cette configuration, point n'est besoin d'attendre la démission du Gouvernement, le président de la République peut aisément renvoyer le Premier ministre, même si dans la pratique la voie de la démission volontaire semble privilégiée ${ }^{70}$. Toutefois, cela n'est pas sans conséquence, notamment en présence d'une cohabitation. Il sera toujours possible, pour le chef de l'État, de congédier le Premier ministre de la majorité parlementaire.

La volonté de lever l'ambiguité sur la répartition des compétences entre le président de la République et son Premier ministre était un véritable fil rouge pour les constituants congolais de 2015. Alors que l'article 20 de la Constitution de 1958 attribue au Gouvernement français le pouvoir de déterminer et conduire la politique de la Nation - confortant au passage la dyarchie -, l'article 99 de la Constitution de 2015 précise que c'est en concertation avec le président de la République que le Premier ministre détermine la politique économique et sociale de la Nation ${ }^{71}$. Quoi de plus logique, puisqu'en réalité, en élisant le président de la République au suffrage universel direct, «le peuple participe aux affaires publiques par le biais de l'élection ${ }^{72}$ ». Par voie de conséquence, il est normal que celui qui est le réceptacle de la confiance populaire participe à la détermination de la politique de la Nation. La précision apportée par le constituant semble donc superfétatoire, puisqu'en choisissant le Premier ministre, le chef de l'État d'une certaine manière choisit celui qui est le plus à même de mettre en scène le programme pour lequel il a été élu. C’est pourquoi, sous la $V^{\mathrm{e}}$ République, la nomination du Premier ministre - sauf en période de cohabitation ${ }^{73}$ - donne une indication sur la manière dont l'élu de

Mollet: un camarade en République, Lille, Presses universitaires du Septentrion, 1987, p. 431.

70. Le 16 août 2017, l'actuel Premier ministre Clément Mouamba avait présenté la démission de son équipe gouvernementale au président de la République, avant d'être reconduit quelques jours plus tard.

71. L'art. 99 de la Constitution congolaise de 2015 prévoit que « le Premier ministre, en concertation avec le président de la République, détermine la politique économique et sociale de la Nation».

72. Isabel Thumerel, «La participation du peuple par l'élection dans les Constitutions de 1791 à 1848 », Jurisdoctoria, $\mathrm{n}^{\circ}$ 4, 2010, p. 17.

73. Christophe de Nantois, «La solidarité gouvernementale sous la $V^{\mathrm{e}}$ République: se soumettre, se démettre ou disparaître», Jus Politicum, $\mathrm{n}^{\circ}{ }_{2}$, http://juspoliticum.com/article/La-solidarite-gouvernementale-sous-la-VemeRepublique-se-soumettre-se-demettre-ou-disparaitre-73.html. 
l'Élysée veut voir formalisé son contrat de confiance passé avec le peuple. Ainsi, la nomination d'Édouard Philippe, membre des Républicains ${ }^{74}$, confirmait la volonté du tout nouveau président Macron d'appliquer une politique pragmatique, débarrassée des influences idéologiques et partisanes $^{75}$. De même, la nomination de Clément Mouamba - alors membre de l'Union panafricaine pour la démocratie sociale (UPADS), parti d'opposition - comme Premier ministre, marquait non seulement un signe d'ouverture vis-à-vis de l'opposition, mais aussi la priorité donnée à la relance économique ${ }^{76}$. Dans les deux cas, nul ne peut douter que l'un et l'autre présidents évaluent les risques d'un désaccord avant de confier les clés du gouvernement à une personnalité.

Les constituants congolais ont tout fait pour annihiler la possibilité d'une cohabitation ravageuse pour le président de la République. Sinon, comment expliquer toutes les précautions pour placer le Premier ministre en infériorité par rapport au président de la République ? Parmi celles-ci, il convient de souligner la précision répétée sur l'encadrement du pouvoir de nomination du chef du Gouvernement. En effet, que ce soit dans l'exercice de son pouvoir réglementaire et d'exécution des lois, ou encore dans la nomination aux emplois civils et militaires (article 101), le constituant a pris soin de préciser que cela se faisait en dehors des actes et nominations pris en conseil de ministres ${ }^{77}$, comme si l'absence d'une telle précision dans la Constitution de 1958 conduisait à un enchevêtrement des compétences entre les deux.

Finalement, à force de vouloir se démarquer de l'originale, le constituant congolais de 2015 a instauré une véritable hiérarchie au sein de l'exécutif. À choisir entre une "présidentialisation monarchique et une présidentialisation dyarchique ${ }^{78}$ ", le choix du constituant s'est porté sur la première option. La différenciation à l'égard du texte de 1958 conduit à un accroissement de la présidentialisation, qui se fait au détriment du Premier ministre et du Parlement.

74. Le 31 octobre 2017, le Conseil national des Républicains a exclu cinq membres du parti, dont le Premier ministre Édouard Phillipe.

75. Décret du 19 juin 2017 portant nomination du Premier ministre.

76. Décret en date du 23 avril 2016.

77. L'art. 101 de la Constitution de 2015 prévoit: «Le Premier ministre assure l'exécution des lois et exerce le pouvoir réglementaire dans les matières autres que celles relevant des décrets en Conseil des ministres. Il nomme aux emplois civils et militaires autres que ceux pourvus en Conseil des ministres ou par décret simple du président de la République.»

78. Daniel Bourmaud, «Les $V^{\text {es }}$ Républiques. Monarchie, dyarchie, polyarchie. Variations autour du pouvoir sous la $V^{\mathrm{e}}$ République», Pouvoirs, vol. 99, n ${ }^{\circ}$ 4, 2001, p. 8. 


\subsubsection{Le Parlement, un souverain sous contrôle}

Le déséquilibre institutionnel de la $\mathrm{V}^{\mathrm{e}}$ République se manifesta de façon éclatante en 1986 , lors de la première cohabitation ${ }^{79}$. Les observateurs du régime découvrirent dès lors l'autre versant de la Cinquième, plus parlementariste, rejoignant à l'unisson la famille des régimes parlementaires. Dans cette configuration, loin du « régime parlementaire à forte domination présidentielle ${ }^{80} »$ de Guy Carcassonne; si l'exécutif demeure le pôle central du régime, cependant, le centre de commandement se déplace de l'Élysée vers Matignon, grâce à la majorité à l'assemblée qui cesse d'être présidentielle pour devenir primoministérielle. La cohabitation c'est donc avant tout l'insubordination du parlement, ou plutôt la revanche d'une institution conçue, sous la $\mathrm{V}^{\mathrm{e}}$, comme un danger pour le pouvoir exécutif ${ }^{81}$.

L'hypothèse d'une volonté du constituant congolais de 2015 de dépasser la $\mathrm{V}^{\mathrm{e}}$ République prend corps dans une série de dispositions concernant le Parlement qui tendent à préserver la prééminence du chef de l'État, même dans le cas où une majorité opposée serait élue à la chambre des députés. Tout d'abord, il convient de souligner qu'en matière parlementaire, l'influence du modèle constitutionnel français est toujours vivace au Congo. Ainsi, dans la nouvelle Constitution, on retrouve l'essentiel des techniques parlementaires de la $\mathrm{V}^{\mathrm{e}}$ République. En premier lieu, le «dépassement définitif de la souveraineté de la loi ${ }^{82}$ » est acté par une "répartition ordinaire des compétences entre la loi et le décret ${ }^{83}{ }^{»}$. Ainsi, l'article 125 pose le domaine de la loi, alors que l'article 126 vient limiter ce domaine, en posant le domaine d'exception qui est celui du règlement. En réalité, la technique consiste à «aménager le statut du Parlement de façon à ce qu'il ne soit plus [ou pas] le centre de la vie politique ${ }^{84} »$. Par conséquent, l'ensemble des

79. Jean-Manuel Larralde, «La réforme de 2008 , une réelle revalorisation du rôle du Parlement ? », CRDF, $\mathrm{n}^{\circ}$ 10, 2012, p. 107.

80. Guy Carcassonne, «Immuable V ${ }^{\mathrm{e}}$ République », Pouvoirs, $\mathrm{n}^{\mathrm{0}}$ 126, 2008, p. 27-35.

81. Jean-Pierre Duprat, «L'influence de la Constitution sur la vie parlementaire», dans Bertrand Mathieu (dir.), op. cit.

82. Valerio Onida, "Dossier $50^{\mathrm{e}}$ anniversaire», Cahiers $d u$ Conseil constitutionnel, $\mathrm{n}^{\mathrm{o}} 25$, août 2009.

83. Georges Galichon, «Aspects de la procédure législative en France », Revue française de science politique, $\mathrm{n}^{\circ} 4,1954$, p. 793.

84. Didier Maus, "La Constitution jugée par sa pratique. Réflexions pour un bilan », Revue française de sciences politiques, op. cit., p. 887. 
techniques d'asservissement du Parlement, pompeusement qualifiées de rationalisations du parlementarisme, sont présentes ${ }^{85}$.

D'abord, en ce qui concerne la fonction de contrôle, le constituant, dans la droite ligne de la $\mathrm{V}^{\mathrm{e}}$ République, recherche en premier lieu la stabilité gouvernementale. De cette façon, la Constitution encadre scrupuleusement la motion de censure prévue par l'article 160, en l'enserrant dans un formalisme strict. La procédure est presque la même que celle prévue par l'article 49 de la Constitution de 1958. Toutefois, le constituant congolais va plus loin, en exigeant la signature d'un quart des députés alors qu'un dixième suffit dans la Constitution française. De même, alors que dans la Constitution de 1958 une majorité suffit pour adopter la motion, la Constitution congolaise de 2015 exige une majorité de deux tiers.

A contrario, de façon inédite dans l'histoire constitutionnelle congolaise ${ }^{86}$, le président de la République dispose de deux moyens pour dissoudre l'Assemblée nationale. D'abord, par l'article 162 qui permet au président de la République de dissoudre l'Assemblée en cas de constat d'un changement de majorité survenue à l'Assemblée nationale ${ }^{87}$.

Ensuite, l'article 165 évoque l'idée d'une crise persistante entre le Gouvernement et l'Assemblée nationale qui conduirait le chef de l'État à dissoudre l'Assemblée ${ }^{88}$. Ces deux articles demandent quelques observations. En effet, si au nom du fonctionnement régulier des pouvoirs

85. Raymond Ferretti, La rationalisation du régime parlementaire sous la Cinquième République, juin 2014, http://ferretti.imingo.net/RATIONALISATION.pdf; JeanMarc Sauvé, «La revalorisation des Parlements? », Journée d'étude organisée par la société de législation comparée, 19 mars 2010, http://www.conseil-etat.fr/content/ download/1709/5155/version/1/file/intervention_revalorisation_parlements.pdf.

86. Dans la Constitution de mars 1992, seul l'art. 8 prévoyait une dissolution de l'Assemblée nationale, en disposant que «lorsque l'équilibre des institutions publiques est rompu notamment en cas de crise aiguë et persistante entre le pouvoir exécutif et le Parlement, ou si l'Assemblée nationale renverse à deux reprises le Gouvernement en l'espace d'un an, le président de la République peut après consultation du Premier ministre et du président de l'Assemblée nationale prononcer la dissolution de l'Assemblée nationale».

87. Art. 162 de la Constitution de 2015: «Le Président de la République, après avoir constaté les changements intervenus à l'Assemblée nationale et après consultation des présidents des chambres du Parlement et du Premier ministre, peut prononcer la dissolution de l'Assemblée nationale. »

88. Art. 165 de la Constitution de 2015: «En cas de crise persistante entre le Gouvernement et l'Assemblée nationale rendant impossible le fonctionnement régulier des institutions, le président de la République, après avoir informé les présidents des deux chambres et le Premier Ministre, prononce la dissolution de l'Assemblée nationale.» 
publics, on peut aisément comprendre que le président de la République dissolve la chambre des députés (article 165), en revanche, il est plus difficile de comprendre pourquoi il organiserait des élections anticipées à la suite d'un changement de majorité survenue au sein de l'Assemblée nationale. Les députés ou les partis politiques sont libres de se reconstituer à l'intérieur de la chambre délibérative, d'ailleurs le mandat impératif est nul (article 128, alinéa 2), et l'article 112 prévoit la déchéance pour les cas de transhumance politique ${ }^{89}$. Dès lors, si regroupement il y a au sein de l'Assemblée nationale, dans l'hypothèse de l'article 162, ce ne sera pas individuel, mais collectif. Il n'y a donc pas de raison objective justifiant l'existence de cet article. Toutefois, seule l'histoire constitutionnelle permet d'expliquer cette «bizarrerie constitutionnelle ${ }^{90} »$. En effet, en 1992, une majorité de circonstances renversa le Gouvernement Bongo-Nouarra, issu de la majorité sortie des urnes, ce qui plongea le pays dans une guerre civile ${ }^{91}$. Toutefois, une pratique inconstitutionnelle des institutions ne peut tout justifier.

Il serait erroné de croire que le parlementarisme en France est demeuré le même depuis 1958. En effet, la prise de conscience de la véracité du «parlement pourquoi faire?», d'André Chandernagor ${ }^{92}$ a conduit progressivement à une prise de conscience sur la nécessité d'une revalorisation de l'institution parlementaire ${ }^{93}$. À partir de là, il ne faut pas se méprendre sur l'influence qu'aurait exercée le parlementarisme français

89. Benjamin Boumakani, «La prohibition de la "transhumance politique" des parlementaires. Étude de cas africains", Revue française de droit constitutionnel, vol. $75, \mathrm{n}^{\mathrm{o}} 3,2008$, p. 499-512. https://www.cairn.info/revue-francaise-de-droitconstitutionnel-2008-3-page-499.htm.

90. Franc de Paul Tetang, «De quelques bizarreries constitutionnelles relatives à la primauté du droit international dans l'ordre juridique interne: la Côte d'Ivoire et l'affaire de l'élection présidentielle», Revue française de droit constitution$n e l$, vol. $91, \mathrm{n}^{\mathrm{o}} 3$, 2012, p. 45-66, https://www.cairn.info/revue-francaise-de-droitconstitutionnel-2012-3-page-45.htm.

91. Patrice Yengo, La guerre civile au Congo-Brazzaville, op. cit., p. 178.

92. André Chandernagor, Un Parlement pourquoi faire?, Paris, Gallimard, 1967.

93. Notons par exemple que pour le comité Vedel, «le Parlement français, et notamment l'Assemblée nationale [était] dans une situation de subordination excessive par rapport au pouvoir exécutif. Cette dernière est d'autant plus regrettable que la profusion normative et la complexité des textes qui marquent depuis près de deux décennies le paysage juridique français ont fait perdre à la loi la qualité qui devrait être la sienne, en même temps que le Parlement a vu se réduire une partie de son prestige et de sa confiance en lui-même» (Rapport remis au président de la République le 15 février 1993 par le comité consultatif pour la révision de la Constitution, JORF, nº 39, 16 février 1993, p. 2544). 
sur le constituant congolais de 2015. De cette manière, alors que les promoteurs de la Constitution de 2015 ont opté pour un "parlementarisme excessivement rationalisé ${ }^{94}$ », en France, peu à peu, par retouche ${ }^{95}$, puis par une réforme de grande ampleur, la $\mathrm{V}^{\mathrm{e}}$ République est devenue un régime parlementaire presque équilibré. Sans passer en revue toutes les procédures du parlementarisme rationalisées en France, on peut noter les écarts entre le système mis en place au Congo qui est celui de 1958, et celui en vigueur en France aujourd'hui, qui est le fruit d'une évolution progressive du parlementarisme. Ainsi, mise à part l'irrecevabilité financière - prévue par l'article $145^{96}$-, l'irrecevabilité pour empiétement sur le domaine réglementaire - prévue par l'article $146^{97}$-, ainsi que la procédure qui permet au Gouvernement de jouer un rôle de régulateur dans les conflits entre les deux chambres - prévue par l'article $150^{98}$-, qui sont demeurés inchangés dans la Constitution de 1958, pour le reste, on note de sensibles évolutions qui malheureusement ne sont pas reprises au Congo. De cette manière, l'article 147 de la Constitution de 2015 prévoit que «la discussion des projets de loi porte, devant la première chambre saisie, sur le texte présenté par le Gouvernement", comme le prévoyait initialement l'article 42 de la Constitution de $1958^{99}$. Cette ancienne version conservée dans la Constitution congolaise est un véritable atout pour le Gouvernement dans la procédure législative, puisqu'elle lui permet, dès lors qu'il ne souhaite pas la transformation de son texte lors de la discussion parlementaire, d'écarter les amendements de ces derniers en faisant usage de cette arme constitutionnelle. Depuis la réforme constitutionnelle française de 2008, c'est désormais sur la

94. Pascal Jan, «Les débats des projets à partir du texte de la commission : disposition innovante... sous conditions ou risque d'un nouveau déséquilibre?», Les Petites Affiches, $\mathrm{n}^{\circ} 254,2008$, p. 68.

95. On peut citer les révisions du n ${ }^{\circ} 92-554$ du 25 juin 1992, instituant le droit de regard parlementaire sur la production européenne; la L. const. $n^{\circ}$ 95-880, 4 août 1995 portant extension du champ d'application du référendum, instituant une session parlementaire unique; la L. const. $\mathrm{n}^{\circ} 96-138,22$ février 1996 créant les lois de financement de la sécurité sociale.

96. L'art. 40 de la Constitution de 1958 prévoit cette irrecevabilité financière.

97. Dans la Constitution de 1958, c'est l'art. 41 qui prévoit l'irrecevabilité pour empiétement au domaine réglementaire.

98. L'art. 45 de la Constitution de 1958 prévoit une disposition similaire.

99. La version antérieure à 2009 disposait: «La discussion des projets de loi porte, devant la première Assemblée saisie, sur le texte présenté par le Gouvernement. Une Assemblée saisie d'un texte voté par l'autre Assemblée délibère sur le texte qui lui est transmis. " 
version issue de la discussion en commission que porte l'examen du projet de loi ${ }^{100}$. Ainsi, Thibaud Cartier-Molin pense que cette disposition devrait encourager le Gouvernement à convaincre les parlementaires lors de la discussion ${ }^{101}$, ce qui ne sera pas le cas au Congo, où l'ancienne version est toujours d'actualité.

Il en est de même, pour la procédure du vote bloqué de l'article 49.3 de la Constitution de 1958, qui permet au Gouvernement de soumettre la chambre des députés. Qualifié d' « instrument emblématique du parlementarisme rationalisé » par la Commission Balladur ${ }^{102}$, il est désormais cantonné à une utilisation exceptionnelle, notamment pour des textes d'une grande importance. Dans ce sens, il n'est désormais utilisable que pour le vote d'une loi de finances, d'une loi de financement de la sécurité sociale ou encore pour toute autre loi, mais qu'une fois par session.

On pourrait également souligner l'extension des pouvoirs du Parlement en France, qui a conduit le constituant organique à créer les lois de programmation, notamment en matière des finances publiques (article 34). C'est aussi le cas, avec la possibilité d'adopter des résolutions, qui sont des prises de position parlementaires.

La volonté du constituant congolais de 2015 de faire du Parlement un " souverain captif ${ }^{103}$ » est claire et pour ce faire, il s'est servi de nombreux instruments qu'offrait le parlementarisme rationalisé version 1958 . Cependant, comme le soulignait Didier Maus, «la fonction parlementaire a un rôle fondamentalement ambigu. Si elle est trop présente et active, elle risque de déboucher sur l'impuissance; si elle se fait trop discrète et résignée, elle encourt la critique de ceux pour qui l'expression "godillot” est péjorative ${ }^{104} »$. Avec une absence de référendum d'initiative parlementaire contrairement à la Constitution de $2002^{105}$ et l'exigence

100. Dans la version issue de la réforme constitutionnelle de 2008 , actuellement en vigueur, l'art. 42 al. 1 dispose: «La discussion des projets et des propositions de loi porte, en séance, sur le texte adopté par la commission saisie en application de l'article 43 ou à défaut, sur le texte dont l'assemblée a été saisie. »

101. Thibaud Cartier-Molin, «La portée du nouveau rôle législatif des commissions parlementaires (commentaires des deux premiers alinéas de l'article 42 de la Constitution)», Revue du droit public, $\mathrm{n}^{\circ}$ 5, 2010, p. 139.

102. Comité de réflexion et de proposition sur la modernisation et le rééquilibrage des institutions de la $\mathrm{V}^{\mathrm{e}}$ République, Une $\mathrm{V}^{e}$ République plus démocratique, proposition, $n^{0} 68$, p. 65.

103. Selon l'expression d'André Tardieu, Le souverain captif, Paris, Flammarion, 1936.

104. Didier Maus, "La Constitution jugée par sa pratique. Réflexions pour un bilan ", art. cité, p. 898 .

105. Aux termes de l’art. 86 de la Constitution de 2015 : «Le président de la République a seul l'initiative du référendum.» 
d'un tiers des députés ou des sénateurs pour saisir la Cour constitutionnelle ${ }^{106}$, c'est sans doute le choix du « godillodisme » qui a été opéré pour les parlementaires congolais.

L'influence du modèle constitutionnel français au Congo mérite d'être nuancée, car si elle est effective, elle se manifeste de trois manières différentes. Il peut s'agir de la reprise totale d'une disposition - quoique la pratique puisse différer -, c'est le cas, par exemple, de l'irrecevabilité financière des parlementaires. L'influence peut également se manifester par la reprise d'une disposition qui n'est plus en vigueur en France, mais répond toujours à une réalité congolaise, c'est le cas de la division des sessions parlementaires. Enfin, elle peut aussi transparaitre dans la reprise d'une disposition, qui sera toutefois tropicalisée, les ordonnances de l'article 153 de la Constitution de 2015 l'illustrent parfaitement ${ }^{107}$.

Au-delà de cette influence incontestable, le constituant de 2015 a voulu colorer son ouvre constitutionnelle d'une couleur plus locale, endogène et répondant à certaines réalités typiquement africaines.

\section{Un bloc constitutionnel identitaire}

Il est aujourd'hui assez aisé de convenir que depuis bientôt trois décennies, nous vivons l'essor de la globalisation, entendue comme une «réunion d'éléments constituant une globalité ». Ce "processus d'uniformisation $^{108}$ " tend à gommer les particularités, conduisant à une standardisation des valeurs et des principes. Eodem tempore, on s'accorde à dire qu'il s'agit aussi du "point d'accélération de la diffusion mondiale du constitutionnalisme $\mathrm{e}^{109} »$. Or, comme le précise Élodie Derdaele, une Constitution, pour être effective, «doit être le reflet

106. L'art. 178 de la Constitution de 2015 prévoit que: «La Cour constitutionnelle est saisie par le Président de la République, le Président de l'Assemblée nationale, le Président du Sénat, le Premier ministre ou par un tiers des membres de chaque chambre du Parlement. »

107. Alors que dans l'art. 38 de la Constitution de 1958, en cas de rejet de la demande d'autorisation, la procédure ne peut continuer; au Congo, il est toujours possible de prendre des ordonnances avec l'aval de la Cour constitutionnelle.

108. Alexandre Viala, "Le concept d'identité constitutionnelle: approche théorique», dans Laurence Burgorgue-Larsen (dir.), L'identité constitutionnelle saisie par les juges en Europe, Paris, Pédone, 2011.

109. Jean Du Bois de Gaudusson, "Défense et illustration du constitutionnalisme en Afrique après quinze ans de pratique du pouvoir», dans Renouveau du droit, Mélanges en l'honneur de Louis Favoreu, Paris, Dalloz, 2007, p. 610. 
de la nation et ainsi être dotée d'une identité propre ${ }^{110}$ », on aboutit inéluctablement à une confrontation entre l'identité propre de l'État qui veut se doter d'une Constitution, et le standard commun des droits et principes portés par la globalisation. De cette manière, si on admet la victoire du modèle occidental de démocratie - conduisant d'ailleurs à «l'occidentalisation du monde ${ }^{111}$ »-, on peut néanmoins souligner certaines résistances ${ }^{112}$ qui s'expliquent par la volonté des identités de ne pas se laisser broyer par le phénomène décrit. Dès lors, on peut facilement déceler dans chaque Constitution cette tension entre la volonté de s'arrimer au train de la globalisation et celle d'affirmer sa propre identité.

La Constitution congolaise de 2015 n'échappe pas à cette tension. Ainsi, au côté d'une prégnance signalée du modèle constitutionnel français, le constituant congolais de 2015 a entendu mettre en évidence un certain nombre de dispositions souvent tirées de la réalité culturelle et politique congolaise. C'est l'ensemble de ses dispositions et de ses institutions que nous qualifions de bloc constitutionnel identitaire, parce que répondant à un souci d'identification de la Constitution. Cependant, il ne suffit pas de désigner un concept ou encore un groupe de mots pour le rendre intelligible, il est indispensable de lui donner un contenu (2.1), avant de voir, en pratique, ce qu'il en est exactement (2.2), et finalement de constater avec Jean-Marie Breton : la "volonté accrue de réinsertion des cadres organiques et des mécanismes fonctionnels du pouvoir dans une vision authentiquement africaine ${ }^{113}$ ».

\subsection{Le cadre conceptuel}

À l'heure de la standardisation des constitutions ${ }^{114}$, il est important de se demander si la loi fondamentale d'un pays peut être un marqueur

110. Élodie Derdaele, «La Constitution entre norme et symbole, réflexions sur le constitutionnalisme contemporain ", VII ${ }^{e}$ Congrès français de droit constitutionnel, Paris, 25-27 septembre 2008 .

111. Yves Laberge et Serge Latouche, L'occidentalisation du monde. Essai sur la signification, la portée et les limites de l'uniformisation planétaire, Paris, La Découverte, 2005, p. 1.

112. Babacar Guèye, "La démocratie en Afrique: succès et résistances", Pouvoirs, $\mathrm{n}^{\mathrm{o}}$ 129, avril 2009, p. 5-26.

113. Jean-Marie Breton, "Trente ans de constitutionnalisme d'importation dans les pays d'Afrique noire francophone entre mimétisme et réception critique: cohérences et incohérences (1960-1990) ", art. cité.

114. Jeremy Mercier, "Sur la standardisation constitutionnelle», IX ${ }^{e}$ Congrès mondial de l'AIDC: les défis constitutionnels globaux et locaux, 16-20 juin 2014, Oslo; 
de son identité. La réponse sera sans doute différente selon l'angle choisi. Ainsi, d'un point de vue sociologique, la Constitution sera pensée comme «le compromis instantané du jeu politique [...] un texte qui ne pourrait rendre compte de la complexité sociale». A contrario, la vision du juriste serait tout autre, "la Constitution, acte juridique suprême, ne saurait être un mode abstrait d'organisation des pouvoirs puisqu'elle puise ses inspirations dans une philosophie politique, dans une histoire nationale et dans la société ${ }^{115}$ ». De ce point de vue, il y a donc une interconnexion entre la Constitution, "norme suprême qui véhicule tout un imaginaire ${ }^{116}$ » et l'identité, entendue comme la spécificité d'une Nation, ce qui fait qu'elle soit différente des autres. Dès lors, l'identification de certaines dispositions constitutionnelles spécifiques à la République congolaise dans la Constitution de 2015 nous conduit à admettre l'existence d'un bloc constitutionnel identitaire, auquel il serait pertinent de définir les contours conceptuels.

\subsubsection{Essai de définition}

Le bloc constitutionnel identitaire représente l'ensemble des dispositions constitutionnelles particulières et spécifiques à un ordre juridique, dont l'existence répond à la nécessité de tropicaliser la norme fondamentale, sans porter atteinte aux principes de la démocratie universelle. Trois traits doivent être mis en avant dans cette définition. D'abord, les dispositions en question sont nécessairement constitutionnelles et particulières à un ordre juridique; ensuite, elles répondent à la nécessité d'une adaptation de la Constitution aux réalités socioculturelles; enfin, elles ne doivent pas porter atteinte aux principes universels de la démocratie.

La première caractéristique du bloc constitutionnel identitaire est donc relative à sa nature. Il s'agit des dispositions d'ordre constitutionnelles, ainsi, elles ne peuvent avoir pour norme de référence que la Constitution. Nous sommes ici au cœur de la fonction symbolique et

Abraham Hervé Diompy, Le paradoxe de l'internationalisation du droit constitutionnel en Afrique: réflexions sur les interactions normatives, institutionnelles et politiques dans l'espace CEDEAO, thèse, université de Bordeaux, 2017.

115. Laurent Sermet, «Les manifestations constitutionnelles de l'identité », Étude Océan Indien, $\mathrm{n}^{\mathrm{o}} 49-50,2013$, p. 1, https://journals.openedition.org/oceanindien/1947 (les deux citations successives).

116. Élodie Derdaele, art. cité. 
intégrative de la Constitution ${ }^{117}$. En effet, si l'on admet historiquement deux critères de définition : le critère formel et le critère matériel ${ }^{118}$, nous convenons néanmoins avec Jörg Gerkrath « que la notion de constitution ne prend toute sa signification que lorsqu'on tient compte des fonctions qui lui sont assignées ${ }^{119}{ }$. Ceci s'explique par le fait que les textes fondamentaux ont été conçus en Occident dans un contexte particulier, celui de la limitation de l'absolutisme. Dès lors, sa première fonction était, et est encore, de garantir les libertés individuelles en limitant le pouvoir des gouvernants ${ }^{120}$. Toutefois, la Constitution assure aussi une fonction intégrative ${ }^{121}$ qui permet aux citoyens d'un État de s'identifier à leur norme fondamentale. Ainsi, dans la Constitution de 2015, deux articles mettent en évidence cette identification, ce sont les articles 5 et 6 : le premier désigne les symboles de l'État, l'emblème national; le second, l'hymne national, les armoiries de la République et même les langues officielles et nationales. Ces articles portent une charge symbolique importante, car il s'agit des symboles de la représentation de l'État. Tous les citoyens congolais se reconnaissent autour de l'hymne national - La Congolaise-, ou encore des couleurs vert, jaune et rouge du drapeau national. La consécration constitutionnelle de deux langues nationales ${ }^{122}$ : le Lingala, parlée au nord du pays et le Munukutuba, usitée au sud du pays, permet à l'ensemble du peuple de s'identifier à la norme suprême qui reconnaît

117. Marie-Claire Ponthoreau, «De la Constitution invisible à la Constitution évanescente, la loi fondamentale de la Hongrie à la lumière des expériences européennes", dans Mélanges en l'honneur de Pierre Bon, Paris, Dalloz, 2014, p. 525-540.

118. Jean-François Aubert (dir.), La Constitution, son contenu, son usage, Recueil des travaux présentés au $125^{\mathrm{e}}$ congrès des juristes suisses, Basel, Helbing \& Lichtenhahn, 1991.

119. Jörg Gerkrath, «Signification et fonctions d'une Constitution», Forum für Politik, Gesellschaft und Kultur in Luxemburg, $\mathrm{n}^{\circ}$ 286, mai 2009, p. 23.

120. Ainsi s'expliquent les deux critères posés dans l'art. 16 de la DDHC: «Toute société dans laquelle la garantie des droits n'est pas assurée, ni la séparation des pouvoirs déterminée, n'a point de Constitution.»

121. Concernant la fonction intégrative des Constitutions dans le cadre des transitions démocratiques, voir Magalie Besse, "L'écriture multilatérale des Constitutions, facteur de réussite des transitions démocratiques », Nouveaux Cahiers du Conseil constitutionnel, $\mathrm{n}^{\mathrm{o}} 45$, octobre 2014.

122. Anne-Marie Le Pourhiet, "Langue(s) et Constitution(s)», Raisons politiques, vol. 2, $\mathrm{n}^{\mathrm{o}} 2$, 2001, p. 207-215, https://www.cairn.info/revue-raisons-politiques2001-2-page-207.htm. Pour une étude spécifiquement africaine, voir Nazam Halaoui, "L'identification des langues dans les Constitutions africaines", Revue française de droit constitutionnel, vol. 45, $\mathrm{n}^{0} 1,2001, \mathrm{p} .31-53$, https://www.cairn.info/revue-francaise-de-droit-constitutionnel-2001-1-page31.htm. 
la spécificité linguistique de chaque zone géographique du pays. De même, la spécificité ou la particularité de telles dispositions ne souffre d'aucune contestation, puisque de manière générale, l'hymne national et le drapeau d'un pays sont souvent exclusifs à celui-ci. Le rapport entre la Constitution et l'identité est ici manifeste, car le lingala, le Munukutuba et La Congolaise sont "des éléments constitutifs de l'identité ${ }^{123}$ ».

La seconde caractéristique du bloc constitutionnel identitaire est la finalité assignée aux dispositions identitaires. En effet, face au processus irréversible de la globalisation du droit en œuvre depuis plusieurs décennies - qualifié de "fétichisme juridico-politique ${ }^{124}$ »-, les États, qui pourtant se plient à cette globalisation, revendiquent toutefois « une identité politique en tant qu'État et République sui generis» ${ }^{125}$. Cette revendication se manifeste par une série de dispositions constitutionnelles, censées rattacher l'État à une histoire, une culture et donc une identité. De cette façon, la Constitution de 2015 prévoit plusieurs dispositions qui à n'en point douter expriment cette revendication identitaire constitutionnelle. Ainsi, la reprise de la Charte de l'unité nationale et la Charte des droits et libertés du 29 mai $1991^{126}$ dans le préambule de la Constitution de 2015 - deux textes issus de la conférence nationale de 1991, fruit du génie national congolais - reflètent bien cette volonté de faire coexister l'universel et le particulier. Il est en effet curieux de juxtaposer tout à la fois: un catalogue de droits à l'image de ce qui existe dans les constitutions allemande ${ }^{127}$ et espagnole ${ }^{128}$ et un catalogue des droits et libertés, qui pour l'essentiel, reprend les dispositions des deux premières, même s'il existe aussi des dispositions plus endogènes. L'idée ici est de rajouter une touche tropicale à la norme suprême, dont la coloration est de plus en plus occidentale. Cette répétition n'a de sens que par sa finalité: la coexistence d'une identité constitutionnelle

123. Selon l'expression utilisée par la circulaire du Premier ministre français du 12 août 1994, relative à l'emploi de la langue française par les agents publics.

124. Maurice Ahanhanzo Glele, "La Constitution ou loi fondamentale», Encyclopédie juridique de l'Afrique, Abidjan, NEA, 1982, t. 1, p. 38 et suiv.

125. Léopold Donfack Sokeng, "Existe-t-il une identité démocratique camerounaise? La spécificité camerounaise à l'épreuve de l'universalité des droits fondamentaux ", Polis, vol. 1, no spécial, février 1996, p. 37.

126. Vivien Romain Manangou, Le cas de la République du Congo : un exemple de régime constitutionnel autoritaire, thèse, université de La Rochelle, 2014, part. 2, titre 1, chap. 1 , sect. $2:$ " La place des droits et libertés dans le préambule de la Constitution de $2002 »$, p. 325-328.

127. Const. Allemande, 23 mai 1949, titre 1, Les droits fondamentaux, chap. 1 à 19.

128. Const. espagnole, 27 décembre 1978, titre 1, Des droits et devoirs fondamentaux, art. 10 à 55. 
propre, à côté de l'identité constitutionnelle "universelle ». Dès lors, on assiste à une coexistence, ou pour parler comme Donfack Sokeng, à « une rencontre entre l'universel et le singulier [...] relativement à la question des droits fondamentaux ${ }^{129} »$. Finalement, la coloration de la norme suprême par des dispositions identitaires conduit à en faire un Janus à deux têtes, ou plutôt à deux identités, sous la forme «d'une complémentarité des différences ${ }^{130}$ ». La complémentarité est le terme capital dans la conciliation.

La dernière caractéristique du bloc constitutionnel identitaire concerne la nécessité pour les dispositions, dont la finalité est de donner à la norme suprême une couleur locale, de ne pas rentrer en collision avec les dispositions de valeur universelle ${ }^{131}$. Tout se passe comme si l'identité constitutionnelle universelle, c'est-à-dire cet ensemble de valeurs et principes de portée universelle, impose au constituant une ligne de conduite dans la rédaction de la Constitution. Ainsi, l'identité constitutionnelle locale ne doit pas annihiler l'effectivité des principes issus de la standardisation du droit. Une telle perspective est loin d'être aisée, en effet, nous savons avec Pierre-François Gonidec que «les catégories classiques s'adaptent mal ou pas du tout à l'Afrique ${ }^{132}$ ». Ce constat incontestablement valable pour les institutions l'est aussi pour certains principes qui se retrouvent souvent en contradiction avec les valeurs et principes issus de la tradition congolaise. Or, l'édifice ne tient que parce que la cohabitation entre l'universel et le particulier est possible. De cette façon, l'incorporation des éléments identitaires passe au second plan et doit respecter l'équilibre global, dans la mesure où la priorité du constituant semble être l'affirmation des valeurs universelles, pour que la Constitution, ainsi rédigée, soit acceptable et acceptée par la communauté internationale. Dès lors, le bloc constitutionnel identitaire vient s'ajouter à un ensemble cohérent de dispositions, majoritairement issues du processus de globalisation du droit. Dans une telle configuration, nous sommes bien en présence d'une « conjoncture

129. Léopold Donfack Sokeng, art. cité, p. 40.

130. Laurent Sermet, art. cité, p. 2.

131. Salah Eddine Berrahou, «La diversité culturelle et les droits fondamentaux : le défi identitaire ", dans Les Droits fondamentaux, Actes des Journées scientifiques tenues à Tunis en octobre 1996, Bruxelles, Bruyant, 1997, p. 256 et suiv.

132. Pierre-François Gonidec, «Esquisse d'une typologie des régimes politiques africains ", Pouvoirs, ${ }^{\circ} 25,1983$, p. 65. 
de fluidité identitaire ${ }^{133}$ ». Cette fluidité identitaire se justifie par le fait d'une cohabitation entre deux identités au sein de la Constitution. De cette manière, bien que «l'identité nationale [soit] inséparable de l'enjeu de domination politique ${ }^{134}$ ", le bloc constitutionnel identitaire ne vient pas remettre en cause l'identité constitutionnelle universelle, au contraire, elle vient la compléter, l'enrichir sur certains aspects plus locaux, rendant la Constitution plus acceptable auprès de ses vrais destinataires: le peuple.

Dans l'ensemble, l'analyse de la Constitution congolaise de 2015 ne laisse entrevoir aucune contrariété entre les dispositions issues de l'identité constitutionnelle universelle avec celles issues du bloc constitutionnel identitaire. Néanmoins, bien que la Constitution demeure la norme suprême, l'architecture normative d'un État contient d'autres normes, c'est le cas de la loi. Ainsi, la vérification d'une absence de contrariété entre le bloc constitutionnel identitaire et les autres dispositions de la Constitution ne suffit pas pour affirmer la parfaite fluidité identitaire. Il convient encore de vérifier si la compatibilité est aussi valable sur les autres normes. À ce niveau, l'entreprise devient plus périlleuse, car il arrive souvent que la loi devienne la norme d'ajustement des gouvernants. En effet, sous la pression des bailleurs de fonds internationaux, les constitutions adoptées comme vitrine de l'État ont souvent une façade libérale, alors que la loi, principale norme de gouvernance, est plus autoritaire. Ainsi, dans la Constitution congolaise de 2015, si l'article 15 pose le principe de l'égalité entre tous les citoyens congolais ; l'article 17 vient préciser cette égalité entre les femmes et les hommes et attribuer à la loi le pouvoir d'assurer une promotion de la femme dans toutes les fonctions politiques, électives et administratives. Une fois actée cette double consécration constitutionnelle, en adéquation avec les principes et valeurs universels auxquels a souscrit le Congo, on se rend compte que sur le plan législatif, à bien des égards, l'égalité demeure une chimère. Par exemple, la notion du chef de famille, avec tous les droits qui y affèrent existe toujours dans le Code de famille congolais. Dans la même lignée, le Code de la famille prévoit le versement d'une dot de la famille de l'époux envers celle de l'épouse. Cette dot contribue à créer une inégalité dans le couple et au sein de la famille entre l'homme et la femme. Finalement, le danger dans une telle entreprise est une adhésion furtive aux principes

133. Selon l'expression de Luc Sindjoun, «Identité nationale et "révision constitutionnelle" du 18 janvier 1996: comment constitutionnalise-t-on le "nous" au Cameroun dans l'État post-unitaire? », Polis, vol. 1, nº spécial, 1996, p. 4.

134. Jacques Chevallier, L'identité politique, Paris, PUF, 1994, p. 5-14. 
de la démocratie libérale. Le bloc constitutionnel identitaire, souvent complémentaire et assimilable aux principes universels, n'est en réalité qu'une façade servant à camoufler la vraie figure du régime, consacrant ainsi un régime des apparences.

Les contours définitionnels du bloc constitutionnel identitaire posés, il convient de délimiter le concept au regard des notions voisines ou proches.

\subsubsection{La délimitation du cadre conceptuel}

Le lien entre l'identité et la Constitution a conduit à l'émergence d'une série de concepts dont le plus commenté est sans doute celui de «l'identité constitutionnelle». En effet, à travers une décision du Conseil constitutionnel français ${ }^{135}$, nous avons découvert que la primauté du droit de l'union pouvait être relativisée, dès lors que ce dernier était contraire à une règle ou un principe inhérent à l'identité constitutionnelle de la France - la seule exception étant que le constituant ait luimême consenti à une telle entorse. Toujours en Europe, la jurisprudence de la Cour constitutionnelle allemande est allée dans le même sens avec l'arrêt sur le traité de Lisbonne en $2009^{136}$, essayant de montrer que l'une des raisons explicatives de l'existence de cette notion serait à rechercher dans «l'idée de défense et de protection de la Constitution ${ }^{137}$ ». Cependant, dans les deux pays la justice constitutionnelle, tout en mettant en avant un concept censé répondre, ou tout du moins limiter l'érosion de la souveraineté consécutive à la construction européenne, n’apporte aucune définition du concept en œuvre. C'est paradoxalement l'article 4 du paragraphe 2 du traité de Lisbonne ${ }^{138}$ qui pose le plus d'éléments susceptibles d'aboutir à une définition matérielle du concept. Ainsi, il en ressort que «l'Union respecte l'égalité des États membres devant les traités ainsi que leur identité nationale, inhérente à leurs structures fondamentales politiques et constitutionnelles, $y$ compris en ce qui concerne l'autonomie locale et régionale». À la lecture de cette disposition communautaire, on peut affirmer, à la suite de Sébastien Martin, qu'il s'agit

135. Cons. const., 27 juillet 2006, Loi relative au droit d'auteur et aux droits voisins dans la société de l'information, $\mathrm{n}^{\circ}$ 2006-540 DC, JORF, 3 août 2006, p. 11541, consid. 19.

136. Cour constitutionnelle, arrêt du 30 juin 2009 sur le Traité de Lisbonne - BverFG, 2 BvE 2/08 vom du 30 juin 2009, pts. 211, 240 et 241.

137. Franz Mayer, «L'identité constitutionnelle dans la jurisprudence constitutionnelle allemande », dans Laurence Burgorgue-Larsen (dir.), op. cit.

138. Il s'agit en réalité du traité sur l'Union européenne (traité de Maastricht), modifié par le traité de Lisbonne, $\mathrm{JO} \mathrm{n}^{\circ} \mathrm{C} \mathrm{83}, 30$ mars 2010. 
d'une "prise en considération sur le plan juridique des particularités spécifiques et intrinsèques des États ${ }^{139}$ ". On connaît la propension du juge administratif français à se muter en juge constitutionnel ${ }^{140}$. Ainsi, dans sa volonté d'interpréter la décision du Conseil constitutionnel, il a estimé que pouvait être considérées comme relevant de l'identité constitutionnelle française, des normes constitutionnelles constitutives d'une exclusivité de l'ordre juridique français, et n'ayant aucune protection équivalente dans l'ordre juridique communautaire ${ }^{141}$. Cependant, malgré cet effort définitionnel, les juges n'ont pas comblé les attentes des observateurs concernant la réalité du concept d'identité constitutionnelle. Tout du moins, il est possible d'affirmer que dans la recherche prétorienne d'une définition au concept, c'est le caractère particulier des dispositions qui est mis en avant pour justifier son attachement à cette catégorie exceptionnelle de dispositions constitutionnelles.

Dès lors, devant cette absence de définition officielle, la question qui se posait était de savoir ce que recouvre réellement le concept d'identité constitutionnelle. Tout en s'interrogeant sur le contenu du concept ${ }^{142}$, la doctrine s'est chargée de définir et d'apporter des éléments de compréhension de celui-ci ${ }^{143}$. Dans ce sens, si certains ont assimilé l'identité constitutionnelle à une supra constitutionnalité ${ }^{144}$, d'autres en revanche, l'ont reprochée d'être une notion trop restrictive et ont

139. Sébastien Martin, «L'identité de l'État dans l'Union européenne: entre "identité nationale" et "identité constitutionnelle" ", Revue française de droit constitutionnel, vol. $91, \mathrm{n}^{\mathrm{o}} 3$, 2012, p. 14.

140. Bernard Stirn, "Constitution et droit administratif», Nouveaux Cahiers du Conseil constitutionnel, $\mathrm{n}^{\mathrm{o}} 37$, octobre 2012. Pour un cas purement africain, voir Célestin Keutcha Tchapnga, «Le juge constitutionnel, juge administratif au Bénin et au Gabon? ", Revue française de droit constitutionnel, vol. $75, \mathrm{n}^{\circ}$ 3, p. 551-583.

141. CE, Ass., 8 février 2007, Société Arcelor Lorraine, $\mathrm{n}^{\mathrm{o}} 287110$.

142. Bertrand Mathieu, «Le droit communautaire fait son entrée au Conseil constitutionnel», LPA, 22 août 2006, spéc, p. 167.

143. Anne Levade, «Quelle identité constitutionnelle nationale préserver face à l'Union européenne? », dans Hélène Gaudin (dir.), L'État membre de l'Union européenne, ADE, vol. 2, 2004, p. 173 et suiv.; Vlad Constantinesco, «La confrontation entre identité constitutionnelle européenne et identités constitutionnelles nationales: convergence ou contradiction? Contrepoint ou hiérarchie? ", Mélanges en l'honneur de Philippe Manin - L'Union européenne: union de droit, unions des droits, Paris, Pédone, 2010, p. 79 et suiv. ; Jean-Christophe Barbato et Jean-Denis Mouton (dir.), Vers la reconnaissance de droits fondamentaux aux États membres de l'Union européenne? Réflexions à partir des notions d'identité et de solidarité, Bruxelles, Bruylant, 2010.

144. Édouard Dubout, «Les règles ou principes inhérents à l'identité constitutionnelle de la France: Une supra constitutionnalité?", Revue française de droit constitutionnel, vol. $83, \mathrm{n}^{\mathrm{o}} 3$, 2010, p. 451-482. 
proposé des concepts plus larges. Soulignons que le lien entre l'identité et la constitution a inspiré dans le passé et continue à inspirer la doctrine. De ce point de vue, d'autres concepts développés par des auteurs comme Jürgen Habermas, Jean-Philippe Derosier, Placide Moudoudou, ou encore James Tully, méritent d'être confrontés au bloc constitutionnel identitaire, afin de mieux borner ses contours conceptuels.

Jean-Philippe Derosier a proposé le concept de «Noyau constitutionnel identitaire», qu'il a défini comme : «un ensemble de principes constitutionnels formant une catégorie spécifique de normes que l'on appelle les limites constitutionnelles à l'intégration européenne : il s'agit de normes qui échappent à toute possibilité de suppression et qui ont un impact sur le processus d'intégration européenne, en interdisant la production d'une norme primaire de l'Union européenne ou en empêchant l'application d'une norme de droit dérivée, malgré le principe de primauté et d'effet direct ${ }^{145}$.» Le noyau constitutionnel identitaire est un concept dans la forme proche du bloc constitutionnel identitaire, et nous a d'ailleurs inspirés dans la sémantique choisie. En revanche, sa finalité est simplement de justifier le refus d'une érosion progressive de la souveraineté des États membres de l'Union européenne. Il s'agit ici «d'une assimilation de l'identité constitutionnelle à la supraconstitutionnalité ${ }^{146} »$. Ainsi, Dominique Rousseau parle de «bouclier de l'identité nationale ${ }^{147} »$. En somme, le noyau constitutionnel identitaire est le symbole de la résistance d'une certaine identité nationale, face à l'intrusion de ce qui pourrait être qualifié d'identité communautaire ou européenne. De ce point de vue, il existe un parallèle avec le constitutionnalisme identitaire porté en Afrique par une certaine doctrine.

Le concept de «constitutionnalisme identitaire» est une invention du professeur Meledje Djedjro ${ }^{148}$, il voulait, par ce groupe de mots, désigner la manipulation ethnique dans le jeu politique dans la plupart des États africains. L'ethnie relevant d'une identité nationale et le jeu politique étant normalement encadré par la constitution, nous sommes

145. Jean-Philippe Derosier, «Le noyau constitutionnel identitaire, frein à l'intégration européenne. Contribution à une étude normativiste et comparée des rapports entre le noyau constitutionnel identitaire et le droit de l'européenne», VII ${ }^{e}$ Congrès de l'AFDC, Nancy, 16-18 juin 2011.

146. Sébastien Martin, art. cité, p. 21.

147. Dominique Rousseau, «L'identité constitutionnelle, bouclier de l'identité nationale ou branche de l'étoile européenne», dans Laurence Burgorgue-Larsen (dir.), op. cit.

148. Francisco Meledje Djedjro, «Le système politique ivoirien dans la géopolitique ouest-africaine», Revue de droit public, $\mathrm{n}^{\circ}$ 3, 2006, p. 708. 
bien dans la problématique du lien ou encore de la confrontation entre la Constitution et l'identité. En revanche, le concept a été repris et coloré différemment par le professeur Moudoudou. Pour ce dernier, «le constitutionnalisme en Afrique», au sens libéral du terme, doit céder la place à un «constitutionnalisme africain » dans lequel l'ethnie sera «au centre de toute réflexion sur l'organisation politique et institutionnelle des États ${ }^{149}$ ». Il s'agit en réalité « d'un courant doctrinal qui appelle à la naissance d'un constitutionnalisme identitaire africain ${ }^{150}$ ». À y voir de plus près, cette revendication pour un constitutionnalisme identitaire est une forme de repli identitaire, un moyen de renouer avec une identité dont personne ne démontre la compatibilité avec le constitutionnalisme libéral. Or, dans le groupe de mots «constitutionnalisme identitaire», il y a d'abord "constitutionnalisme», et ce concept à une histoire, un sens, celui de la limitation de l'absolutisme. Quel sera donc le sens d'un constitutionnalisme identitaire africain? La première difficulté est clairement sémantique. Au demeurant, le constitutionnalisme identitaire à la sauce africaine est proche du noyau constitutionnel identitaire, en ce que la finalité des deux concepts est une restriction. D’un côté, ériger un mur contre les intrusions du droit dérivé communautaire et de l'autre, construire un constitutionnalisme érigé sur les ferments identitaires authentiquement africains.

$\mathrm{Vu}$ sous cet angle, le constitutionnalisme identitaire est assimilable au « constitutionnalisme de diversité », prôné par James Tully ${ }^{151}$. Pour ce dernier, «le constitutionnalisme moderne met en place un cadre qui s'oppose à la reconnaissance de la diversité culturelle ${ }^{152}$ ». Dans les deux cas, le constitutionnalisme devient l'instrument d'une promotion ou encore d'une reconnaissance culturelle au lieu d'être un moyen pour lutter contre l'absolutisme.

$\mathrm{Au}$ contraire, le bloc constitutionnel identitaire n'a pas pour finalité la survivance d'une hypothétique souveraineté, il vise une complémentarité, une cohabitation entre une identité locale, devenue subsidiaire, et une identité standard, devenue fondamentale. De ce point de vue, il est assez proche du patriotisme constitutionnel tel que le précise Dominique

149. Placide Moudoudou, «L'État africain...», art. cité, p. 116.

150. Koffi Ahadzi Nonou, "Préface», dans Placide Moudoudou, La Constitution en Afrique, Paris, l'Harmattan, 2012, p. 12.

151. James Tully, Une étrange multiplicité. Le constitutionnalisme à une époque de diversité, Bordeaux, Presses universitaires de Bordeaux, 1999.

152. France Houle, «Présentation de l'ouvrage de James Tully, Une étrange multiplicité. Le constitutionnalisme à une époque de diversité », Politique et sociétés, $\mathrm{n}^{\circ}$ 201, 2001, p. 198-201. 
Leydet: «il ne s'agit pas de choisir entre les principes universalistes et une identité culturelle particulière, mais de penser leur complémentarité $^{153}$.»

Finalement, le bloc constitutionnel identitaire désigne un savant dosage entre une adhésion aux principes du constitutionnalisme libéral et la survivance de certains principes identitaires. Cette conciliation est palpable dans la Constitution congolaise de 2015. L'on pourrait aussi dire que la volonté du constituant camerounais de 2016, en promouvant l'autochtonie, était de valoriser un bloc des dispositions constitutionnelles censées représenter l'identité camerounaise aux côtés des principes démocratiques universellement consacrés.

En fin de compte, l'existence d'un bloc constitutionnel identitaire dans les textes fondamentaux du continent est une réponse à la critique sur le caractère uniquement mimétique des constitutions africaines. De cette manière, tout en assurant une totale adhésion à la démocratie universelle, les constituants africains n'omettent pas d'inscrire certaines dispositions qui rappellent les particularités du pays. Pour ainsi dire, qu'à l'épreuve du constitutionnalisme, une certaine identité africaine subsiste pour autant qu'elle soit compatible avec ce dernier.

\subsection{La manifestation de l'identité dans la Constitution de 2015}

Le préambule de la Constitution de 2015 pose «l'impérieuse nécessité de concilier les valeurs universelles de la démocratie et les réalités politiques, sociales et culturelles nationales »; ce qui justifie la coexistence du bloc constitutionnel identitaire aux côtés des principes universels du constitutionnalisme au sein du texte fondamental. Cependant, les valeurs, ou pour ainsi dire l'identité dont il est question ici, c'est l'identité congolaise, et non des identités ethniques. En effet, contrairement aux partisans du constitutionnalisme identitaire qui prônent «l'urgence d'une prise en compte juridique du fait identitaire [ethnique] ${ }^{154} »$, le constituant congolais de 2015, dans la lignée de ses prédécesseurs ${ }^{155}$, a conçu une identité structurée autour d'un projet unitaire. En même temps, il s'est distingué par une innovation institutionnelle pour donner corps à une certaine identité institutionnelle congolaise.

153. Dominique Leydet, "Patriotisme constitutionnel et identité nationale», Études philosophiques, $\mathrm{n}^{\circ}$ 192, 1992, p. 98 et suiv.

154. Guy Rossatanga-Rignault, «Identités et démocratie en Afrique. Entre hypocrisie et faits têtus ", Afrique contemporaine, vol. 242, $\mathrm{n}^{\mathrm{0}}$ 2, 2012, p. 64 .

155. Les constituants de 1992 et 2002 ont toujours fait le choix d'une République unitaire, réfutant toute idée de reconnaissance constitutionnelle de l'ethnie. 


\subsubsection{Le refus constitutionnel de l'État ethnique}

De façon incontestable, le constituant de 2015 a fait de l'unité de la nation congolaise un principe incontournable. En lisant la Constitution de 2015, on ne peut être que surpris par le grand nombre de dispositions rappelant le caractère unitaire de l'État et du peuple congolais, comme si le danger d'une dislocation ethnique guettait le pays. Si de façon presque évidente, l'article $1^{\mathrm{er}}$ rappelle le caractère «souverain, unitaire et indivisible »; l'article 15 pose quant à lui le principe de l'égalité et de la non-discrimination entre tous les citoyens congolais. Dans le même temps, l'article 13 met en garde contre « toute propagande ou incitation à la haine ethnique ». Il est cependant vrai que l'on entend encore dans les rues du Congo des insultes à caractère ethnique, sans que quiconque soit inquiété. Concernant les partis politiques, l'article 58 , leur interdit toute identification à un département, une commune, une religion ou encore à une ethnie. Il faut dire qu'à la fin de la conférence nationale souveraine de 1991, les partis politiques créés semblaient être des porte-étendards régionaux, au lieu d'être des lieux de cristallisation du débat politique ${ }^{156}$. D'autre part, si la liberté de manifestation est bien entendu consacrée, l'article 51 l'interdit dès lors qu'elle a un caractère ethnique.

Devant cette litanie de précautions constitutionnelles évitant l'État ethnique, deux dispositions semblent faire entendre une musique différente. Il s'agit des articles 16 et 28 . Le premier tend à garantir et à promouvoir la protection des peuples autochtones. Une telle reconnaissance peut soulever des questions sur l'unité du peuple congolais, existerait-il un peuple autochtone, au côté du peuple congolais? Ou encore, sommes-nous en présence d'une remise en cause de l'unicité $\mathrm{du}$ peuple congolais? Nous pensons pour notre part que cette reconnaissance de l'autochtonie dans la Constitution n'a en réalité qu'une finalité matérielle, il s'agit de justifier une éventuelle politique législative préférentielle à l'égard des peuples autochtones, souvent tenus à l'écart des retombées de la croissance économique. Nous ne sommes donc pas en présence d'une exaltation du fait ethnique.

Quant à l'article 28 qui reconnaît l'identité culturelle de chaque citoyen, il peut donner l'impression de tendre vers une reconnaissance de la multiculturalité congolaise, ce qui coïnciderait avec une reconnaissance tacite des "peuples congolais». Toutefois, le constituant semble

156. Xavier Kitsimbou, «La démocratie et les réalités ethniques au Congo », thèse de science politique, université Nancy II, 2006; Brice Arsène Mankou, «Le tribalisme», Le Portique, ${ }^{\circ}$ 5, 2007, http://leportique.revues.org/1404. 
pressentir un danger, et apporte aussitôt une limite, en arguant que l'exercice de ce droit ne pourrait se faire en portant préjudice, notamment à l'unité nationale. Par voie de conséquence, si l'identité culturelle de chaque citoyen congolais n'est pas proscrite, en revanche, elle ne peut s'exprimer que si elle est compatible avec l'unité nationale, autrement dit l'identité congolaise.

Le choix d'une surexposition de l'État unitaire, bien qu'assez classique au Congo, peut étonner, eu égard aux positions portées par le $\mathrm{P}^{\mathrm{r}}$ Moudoudou, président de la Commission chargée de rédiger la Constitution. Celui-ci appelle à une «requalification de la forme juridique de l'État africain ", comprendre, ce qu'il qualifie de "réalisme ethnique ", c'est-à-dire, la prise en compte, voire la reconnaissance constitutionnelle du caractère multiethnique des États africains. Ce « réalisme ethnique » devrait conduire, selon lui, à «l'institutionnalisation du dosage ethnique $^{157}$ », entendu comme une norme de gouvernance ${ }^{158}$, pouvant par exemple justifier «la distribution des postes en fonction des critères ethniques ou religieux ${ }^{159}$. Or, à la lecture de la Constitution de 2015 , on est fort heureusement bien loin d'une telle option. Plusieurs raisons à notre avis plaidaient contre l'instauration de l'État ethnique.

D'abord, à en croire le professeur Moudoudou, il conviendrait de permettre "aux diverses régions de s'organiser autour de leurs langues et assises territoriales spécifiques dans le respect de leur identité culturelle et ethnique ${ }^{160} »$. Une telle idée, dans le cadre du Congo, semble impossible, à moins d'envisager l'instauration d'une région par ethnie. Construire des entités administratives sur la seule base ethnique s'est prendre le risque d'un démantèlement permanent, car l'ethnie est par nature dynamique et mouvante. De même, il ne s'agit que «d'un mode de mobilisation politique parmi d'autres et, pas plus qu'ailleurs, nul individu n'y soit réductible à une appartenance identitaire primordiale ${ }^{161}$ ». Ainsi, que deviendra-t-il d'une région conçue à l'origine sur une base ethnique et dont les ressorts de la construction se disloquent avec le

157. Placide Moudoudou, "L’État africain...», art. cité, successivement p. 127 et 133-134.

158. Il faut aussi souligner que d'autres auteurs ont proposé l'institutionnalisation du dosage ethnique, à l'instar du doyen Koffi Ahadzi Nonou, «Les nouvelles tendances...», art. cité, p. 92.

159. Placide Moudoudou «L'État africain... », art. cité, p. 134.

160. Ibid.

161. René Otayek, "La démocratie entre mobilisations identitaires et besoin d'État: y a-t-il une "exception" africaine?», Autrepart. Cahiers des sciences humaines, nouv. série, $\mathrm{n}^{\mathrm{0}}$ 10, 1999, p. 9. 
temps? C'est notamment le cas de la région de la Bouenza, qui avant les indépendances faisait partie de la région du Pool, dans laquelle cohabitaient des populations Kongo. Dans les années 1950, les affrontements avaient opposé ces populations avec des ressortissants du nord du pays, mais dans les années 1990, le bloc Kongo s'est fracturé en deux: d'un côté, les ressortissants du Pool, et de l'autre ceux des régions du Niari, de la Bouenza et de la Lekoumou (Nibolek). Dès lors, comment assurer la pérennité d'une telle entité sans assister à un embrasement ethnique?

Ensuite, le dosage ethnique, censé assurer l'équilibre communautaire dans la gestion de la chose publique, bien que séduisant à première vue, n'est pas la garantie tout risque contre le déchirement communautaire. En effet, le principe est connu et s'applique déjà dans certains pays sans pour autant pacifier les relations intercommunautaires. Ainsi, l'article 16 de la Constitution du Burundi de 2010 impose une forme de dosage ethnique dans la composition du Gouvernement ${ }^{162}$. Pour autant, depuis quelques années, ce pays est au bord de l'éclatement ethnique. Et que dire du Liban dans lequel l'article 25 de la Constitution pose expressément l'obligation d'un dosage ethnique dans la composition de l'Assemblée nationale ${ }^{163}$. Pourtant, les deux pays sont malheureusement placés dans la liste des pays les plus instables, avec des risques importants d'implosion ethnique. Le dosage ethnique n'est donc pas la réponse appropriée pour faire cohabiter les ethnies au sein d'une nation.

Enfin, le doyen Moudoudou semble porter « une conception figée de l'ethnie [y voyant] un état que définit un ensemble de traits invariants

162. Art. 16 de la Constitution du Burundi de 2010: «Le Gouvernement burundais doit être composé de sorte que tous les Burundais y soient représentés et qu'il les représente tous; que chacun ait des chances égales d'en faire partie; que tous les citoyens aient accès aux services publics et que les décisions et les actions du Gouvernement recueillent le plus large soutien possible.»

163. Art. 24 de la Constitution du Liban de mai 1926, modifié notamment en 1927 : «La Chambre des députés est composée de membres élus dont le nombre et les modalités d'élection seront déterminés par les lois électorales en vigueur. En attendant l'élaboration par la Chambre des députés d'une loi électorale sans contrainte confessionnelle, les sièges parlementaires seront répartis conformément aux règles suivantes: a) À égalité entre chrétiens et musulmans; b) Proportionnellement entre les communautés de chacune de ces deux catégories ; c) Proportionnellement entre les régions. / À titre exceptionnel, et pour une seule fois, les sièges parlementaires vacants à la date de la publication de la présente loi ainsi que les sièges qui seront créés par la loi électorale, en application du principe de l'égalité entre chrétiens et musulmans, conformément à la Charte d'entente nationale, seront pourvus par nomination en une seule fois par le Gouvernement d'Union Nationale à la majorité des deux tiers. La loi électorale déterminera les modalités d'application de cet article.» 
comme la culture, les liens de sang ou les affinités naturelles ${ }^{164} »$. Une telle conception, comme l'a démontré Jean-François Bayart, repose sur trois postulats. En premier lieu, la culture serait «un corpus de représentations stables dans le temps "; en second lieu, elle serait "close sur elle-même»; enfin, elle déterminerait "une orientation politique précise $^{165} »$. Cette conception a été remise en cause de façon magistrale par Fredrik Barth. Selon lui, «l'erreur principale de ses prédécesseurs a été de s'intéresser au contenu culturel (point de vue essentialiste ou substantialiste) des catégories, alors qu'il aurait mieux valu étudier les frontières (boundaries) de ces entités, là où se joue le maintien de l'identité ethnique dans les interactions entre membres de groupes différents » ${ }^{166}$. Autrement dit, "l'identité ethnique chez Barth est avant tout le fait d'une auto-attribution, une attribution par les acteurs euxmêmes qui choisissent d'endosser telle ou telle identité ${ }^{167}$ ».

De ce point de vue, il n'est pas tout à fait judicieux d'expliquer l'échec du constitutionnalisme en Afrique par l'absence d'une prise en compte de l'identité ethnique. Dans la mesure où cette dernière n'est qu'une construction collective et circonstancielle.

Pour notre part, nous postulons l'émergence d'un patriotisme constitutionnel, qui se définirait comme "une adhésion essentiellement rationnelle aux principes contenus dans la norme suprême du pays ${ }^{168}$ ». Le patriotisme constitutionnel tel que le défend Habermas est donc « une adhésion aux principes universalistes sous-tendant l'État de droit, par opposition au sentiment particulariste d'appartenance à la nation comme entité historique concrète ${ }^{169}$ ". L'idée ici, comme tente de le faire le constituant de 2015, serait de "concilier l'universalité du cadre juridique préconisé dans la théorie du patriotisme constitutionnel avec la prise en compte des particularités culturelles nationales ${ }^{170} »$. C'est dans ce sens qu'il convient d'expliquer la multiplication d'institutions inédites dans le texte constitutionnel de 2015.

164. René Otayek, art. cité, p. 6.

165. Jean-François Bayart, L'illusion identitaire, Paris, Fayard, 1996, p. 74.

166. Fredrik Barth, «Les groupes ethniques et leurs frontières », dans Philippe Poutignat et Jocelyne Streiff-Fenart, Théories de l'ethnicité, Paris, PUF, 1999, p. 213.

167. Paul Costey, "Les catégories ethniques selon F. Barth», Traces. Revue de sciences humaines, $\mathrm{n}^{\circ}$ 10, 2006, http://traces.revues.org/155.

168. Muriel Rambour, «Le patriotisme constitutionnel, un modèle alternatif d'élaboration d'une identité européenne?», Études européennes, mars 2006, p. 2.

169. Michel Coutu, «Citoyenneté et légitimité. Le patriotisme constitutionnel comme fondement de la référence identitaire», Droit et société, n ${ }^{\circ} 40,1998$, p. 631.

170. Muriel Rambour, art. cité, p. 5. 


\subsubsection{Une diversité institutionnelle assurant l'intégration de l'identité congolaise au sein de la Constitution}

Les concepteurs de la Constitution de 2015 avaient promis que l'initiative du changement visait la modification de la gouvernance ${ }^{171}$; une telle orientation n'était possible qu'en mettant en place de nouvelles institutions susceptibles de garantir cette nouvelle gouvernance. De cette manière, on peut distinguer deux catégories "d'institutions de l'État qui trouvent leur fondement dans la constitution [...] il s'agit non seulement des organes traditionnels de l'État: Chef de l'État, Gouvernement, Parlement ${ }^{172}$ ", mais aussi d'autres institutions qui permettent de mieux rattacher la Constitution aux réalités locales. C'est dans cette dernière catégorie que l'on retrouve la manifestation de l'identité constitutionnelle qu'essaie de mettre en évidence le constituant. En effet, si l'on peut aisément constater le classicisme concernant les institutions concernées par la séparation des pouvoirs - entendue comme "une technique constitutionnelle destinée à éviter le despotisme et à garantir la liberté ${ }^{173} »-$ les autres institutions sont des innovations et relèvent véritablement du génie congolais. À ce titre, elles méritent qu'on s'y attarde.

Le titre XIX de la Constitution de 2015 est consacré aux « conseils nationaux consultatifs ». Bien qu'il s'agisse simplement « d'organes consultatifs », c'est-à-dire des institutions sans pouvoir de contrainte, néanmoins, elles répondent à un souci d'ouverture de la décision politique aux citoyens. En effet, nous savons avec Michael Walzer qu' il existe " des sphères de justice distinctes, correspondant chacune à une conception particulière d'un type de bien entretenue au sein d'une communauté donnée, et relevant de critères de distribution spécifiques. Ce qui vaut dans la sphère économique ne se laisse pas transférer dans la sphère de l'éducation, ou dans celle du pouvoir politique ${ }^{174} »$. Ainsi, il est indispensable de créer des espaces de concertation entre les différentes sphères. Les différentes institutions consultatives créées par la Constitution de 2015 tendent vers cet objectif.

171. Voir la conférence de presse de Thierry Moungalla, le 25 septembre 2015.

172. Christine Desouches et Gérard Conac, «Les institutions constitutionnelles des États d'Afrique francophone et de la République malgache», Politique étrangère, $\mathrm{n}^{\mathrm{o}} 1,1980$, p. 250-252.

173. Francis Hamon et Michel Troper, Droit constitutionnel, Paris, LGDJ, 2016, p. 99.

174. Michael Walzer, Sphères de justice. Une défense du pluralisme et de l'égalité, Paris, Seuil, 2013. 
Il en est ainsi du Conseil consultatif des femmes, dont l'article 232 nous apprend que sa mission sera de donner des avis consultatifs sur la condition des femmes. Cette institution aura surtout pour mission de rendre effectif l'article 17 de la Constitution, qui réaffirme l'égalité entre la femme et l'homme et oblige la loi à garantir la parité, la promotion et la représentativité des femmes à toutes les fonctions politiques.

Quant à l'article 234, il institue le Conseil consultatif des personnes vivant avec handicap, son objectif est de faire des suggestions au Gouvernement pour améliorer la prise en charge des populations concernées.

Dans cette frénésie institutionnelle, la jeunesse et la société civile n'ont pas été oubliées. L'article 236 met en place un Conseil consultatif de la jeunesse, sa mission est d'être le porte-parole de la jeunesse auprès des pouvoirs publics. Dans un pays où la jeunesse représente plus de $70 \% \mathrm{de}$ la population, les défis d'une telle institution seront énormes.

Enfin, l'article 238 crée le Conseil consultatif des ONG et de la société civile, sans doute l'organe le plus important en matière de contrepouvoir $^{175}$.

Cependant, si ce paysage montre une volonté incontestable de coller la constitution aux réalités locales, il n'en demeure pas moins que les questions se posent sur l'effectivité des institutions ainsi mises en place. On se souvient encore de l'inertie des institutions comme la Commission des droits de l'homme ou le médiateur de la République dans la Constitution du 20 janvier 2002. Cette inquiétude est renforcée par le fait que la Constitution renvoie à la loi, notamment la loi organique, pour fixer le régime desdites institutions. Et à l'heure des restrictions budgétaires, il est facile pour la majorité parlementaire de faire semblant d'oublier la mise en place des institutions constitutionnelles.

Toutefois, ces inquiétudes ne semblent pas justifiées pour le Conseil national du dialogue, dont les articles 227 à 229 posent le régime. En effet, le préambule du texte fondamental «affirme son attachement aux vertus du dialogue comme moyen de règlement pacifique des différends dans le cadre d'une République apaisée». La concrétisation de cette orientation supposait la mise en place d'un cadre de concertation, permettant le dialogue permanent entre les forces vives de la nation congolaise. Au demeurant, la contestation populaire et politique de la procédure ayant abouti au changement de constitution, avec une cristallisation du débat

175. Vivien Romain Manangou, "Contre-pouvoirs, tiers pouvoirs et démocratie en Afrique", IX Congrès français de droit constitutionnel, 26-28 juin 2014, http://www.droitconstitutionnel.org/congresLyon/CommLD/D-manangou_T2.pdf. 
autour de la constitutionnalité et la légitimité de la procédure, explique sans doute la nécessité de cette institution.

Il faut dire que, contrairement aux autres institutions constitutionnelles consultatives, celle relative au dialogue a vu son installation se réaliser aux premières heures de la nouvelle République. Ainsi, la loi organique du 7 août 2017 a été adoptée pour rendre effectif le dialogue politique ${ }^{176}$. La lecture de celle-ci soulève quelques interrogations. D’abord, elle se veut un instrument du président de la République; ainsi, les articles $1^{\text {er }}$ et 3 nous apprennent que le dialogue politique se réunit uniquement à la demande du chef de l'État, et qu'il est placé sous son autorité. Or, une telle configuration en présence d'un "présidentialisme paternaliste» ne pourra pas permettre un véritable dialogue entre l'ensemble des protagonistes. Par ailleurs, sa composition pose également problème: si l'article 4 de la loi organique établit une liste des membres de droit - qui devraient pour la plupart d'entre eux être de la majorité présidentielle, eu égard à leur mode de désignation -, l'article 5 accorde au chef de l'État la possibilité d'en nommer d'autres à sa convenance. Nous sommes donc bien en présence d'un instrument de la puissance présidentielle, qui contribuera à renforcer la prédominance de ce dernier sur le fonctionnement des institutions.

À l'instar des Commissions électorales indépendantes, le conseil consultatif pour le dialogue politique est une institution qui pourrait devenir un patrimoine constitutionnel commun en Afrique, tant il est vrai que dans cette partie du monde, les rapports politiques sont toujours extrêmement conflictuels. Bien avant le Congo, au Niger, un Conseil national de dialogue politique (CNDP), défini comme un cadre permanent de prévention, de règlement et de gestion des conflits politiques à travers le dialogue et la concertation entre la classe politique nigérienne, avait été institué par un décret du 30 janvier $2004{ }^{177}$. Pour autant, le bilan de cette commission reste mitigé ${ }^{178}$.

Docteur en droit; Assistant à l'université Marien-Ngouabi; Chargé de communication du PTR CAMES «Gouvernance et Développement »; Membre associé au Centre des études juridiques environnementales et politiques (CEJEP), La Rochelle.

176. L. org. $\mathrm{n}^{\mathrm{o}}$ 30-2017 du 7 août 2017.

177. Décret $n^{\circ}$ 2004-030/PRN/PM, 30 janv. 2004.

178. «(Bilan 2016) Niger/politique: Mahamadou Issoufou reconduit, l'opposition mutilée », Réseau international, 25 décembre 2016, http://reseauinternational.net/ bilan-2016-nigerpolitique-mahamadou-issoufou-reconduit-loppositionmutilee/. 